\title{
Heteroclinic dynamics in a model of Faraday waves in a square container
}

\author{
Jeff Moehlis ${ }^{\mathrm{a}, *}$, Jeff Porter ${ }^{\mathrm{b}}$, Edgar Knobloch ${ }^{\mathrm{c}}$
}

\begin{abstract}
A B S T R A C T
We study periodic orbits associated with heteroclinic bifurcations in a model of the Faraday system for containers with square cross-section and single-frequency forcing. These periodic orbits correspond to quasiperiodic surface waves in the physical system. The heteroclinic bifurcations are related to a continuum of heteroclinic connections in the integrable Hamiltonian limit, some of which persist in the presence of small damping. The dynamics in the neighborhood of one of the heteroclinic bifurcations are examined in detail using approximate Poincare maps, with predictions that agree with numerical computations. The results suggest a great richness of possible dynamics of Faraday waves even in simple geometries and with single-fiequency forcing.
\end{abstract}

\section{Introduction}

The instability by which gravity-capillary waves are excited on the free surface of a viscous fluid through periodic vibration of the container in the vertical is known as the Faraday instability [1]. ln most cases, the first waves to appear are periodic subharmonic standing waves with a period equal to twice the forcing period. These surface waves may settle into a simple pattern (squares or hexagons, for example), or exhibit more complex behavior, depending largely on the influence of mode interactions. [n small aspect ratio systems, the excited modes (and hence the asymptotic behavior of the system) are primarily selected by the shape of the container [2-5]. In large aspect ratio systems, mode competition is more easily generated (and controlled) by the use of multifrequency forcing [6]. A number of experiments (see, e.g. [7-10]) have used such multi-frequency forcing to produce novel patterns including superlattice patterns, quasipatterns, and oscillons,

Even in the classic case of single-frequency forcing, a variety of intriguing dynamics is possible, and much remains to be understood, particularly with regard to container geometry and mode interactions. Experimentally, Ciliberto and Gollub [2] investigated the competition between two nondegenerate modes (i.e., modes unrelated by symmetry) in a container with circular cross-section, and found that chaotic waves may result. In order to achieve the desired mode competition both the forcing frequency and amplitude had to be tuned carefully. Mode competition occurs somewhat more easily in containers of square cross-section because all modes occur in pairs, related by a reflection through the diagonal. Simonelli and Gollub [3] (hereafter SG) considered the interaction between the $(3,2)$ and $(2,3)$ modes for a container with square cross-section, and observed only standing waves as attractors. Occasional quasiperiodic and chaotic time dependence was also found, but this was dismissed as the result of nonsquare imperfections in the container, In this paper we show that quasiperiodic and chaotic waves can in fact occur in a model of the SG experiment in a perfectly square container, and that such waves are associated with heteroclinic bifurcations in the model equations. We remark that quasiperiodic and chaotic surface waves have also been found in a model of a different mode interaction, involving the $(1,0)$ and $(0,1)$ modes, also for a container with square cross-section [4]. That model is of the same form as the one considered in this paper, but with different coefficient values. It is shown in [4] that one branch of quasiperiodic surface waves arises via a Hopf bifurcation, but other bifurcations of quasiperiodic surface wave solutions are not characterized in detail. For completeness, we mention that SG also considered the effect of making the container slightly rectangular. Here the degeneracy between the modes is broken, and quasiperiodic and chaotic surface waves are present near onset.

In this paper we focus on the Faraday instability for containers with perfectly square cross-section. We use the model derived in Feng and Sethna [11] (hereafter FS) and described in Section 2, which includes a linear damping term to account phenomenologically for dissipation in the system [12]. In Section 3, we consider 
a

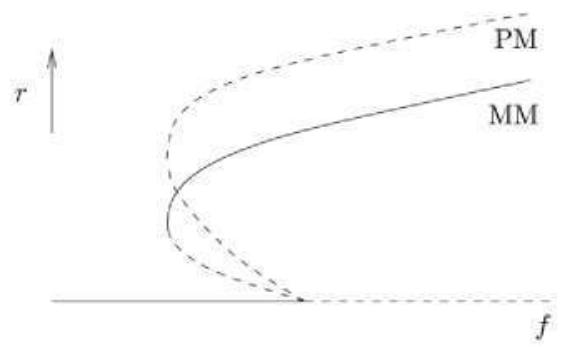

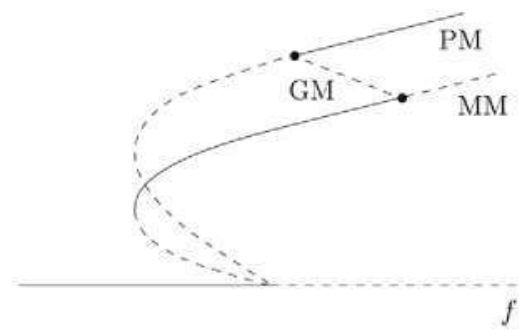

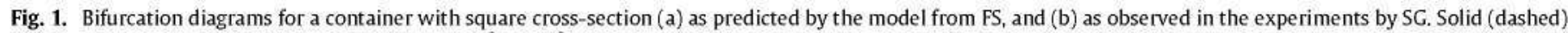
lines denote stable (unstable) solutions, and $r \equiv\left|z_{1}\right|^{2}+\left|z_{2}\right|^{2}$. The model predicts that the stable solutions for large $f$ are MM, but the experiments find these to be PM.

parameters based on the experiments described in SG, and numerically locate various stable periodic orbits in this model; these correspond to stable quasiperiodic surface waves, and persist over a range of damping strengths. Such waves were not found in previous theoretical studies of the SG experiment $[5,13]$ and resemble the quasiperiodic waves found but dismissed by SG. These waves terminate in heteroclinic bifurcations, which are themselves related to a continuum of heteroclinic connections belonging to the integrable Hamiltonian limit, discussed in Section 4. The persistence of such heteroclinic connections in the damped, forced problem is considered in Section 5, while the dynamics in the neighborhood of a specific heteroclinic bifurcation are examined using approximate Poincaré maps in Section 6. Concluding remarks are given in Section 7.

\section{Amplitude equations}

We consider a fluid in an open container of square cross-section, with sides of length $L$, containing a fluid of depth $H$. The container is vibrated vertically with frequency $2 \Omega$, and the forcing amplitude is assumed to be $O(\epsilon)$, where $\epsilon \ll 1$, comparable to the damping and detuning. Following FS, if the flow is taken to be irrotational and incompressible, and the effects of surface tension ignored, then near onset the velocity potential can be expanded in terms of two interacting modes in the form

$$
\begin{aligned}
\phi \sim & \epsilon^{1 / 2} g(z)\left\{z_{1}(\tau) \cos \left(\frac{n \pi x}{L}\right) \cos \left(\frac{m \pi y}{L}\right)\right. \\
& \left.+z_{2}(\tau) \cos \left(\frac{m \pi x}{L}\right) \cos \left(\frac{n \pi y}{L}\right)\right\} \mathrm{e}^{\mathrm{i} \Omega t}+\text { c.c. }+O(\epsilon),
\end{aligned}
$$

where $z_{1}$ and $z_{2}$ are the (complex) amplitudes of the two modes, $g(z)$ is the common vertical eigenfunction and $\tau$ is a slow time $(\tau \sim \epsilon t)$. This ansatz leads to the amplitude equations (see FS)

$\dot{z}_{1}=(\lambda+\mathrm{i} \omega) z_{1}+f \bar{z}_{1}+\mathrm{i} A\left(\left|z_{1}\right|^{2}+\left|z_{2}\right|^{2}\right) z_{1}+\mathrm{i} B\left|z_{1}\right|^{2} z_{1}+\mathrm{i} C \bar{z}_{1} z_{2}^{2}$,

$\dot{z}_{2}=(\lambda+\mathrm{i} \omega) z_{2}+f \bar{z}_{2}+\mathrm{i} A\left(\left|z_{1}\right|^{2}+\left|z_{2}\right|^{2}\right) z_{2}+\mathrm{i} B\left|z_{2}\right|^{2} z_{2}+\mathrm{i} C \bar{z}_{2} z_{1}^{2}$.

In these equations all terms are formally of order one, and all $O\left(\epsilon^{1 / 2}\right)$ corrections have been omitted. Thus $f$ is the scaled strength of the forcing (which may without loss of generality be taken to be real and positive), $\omega$ is the scaled detuning $(\omega>0$ implies that the system is being driven at a frequency less than twice the natural frequency of the excited modes) while $\lambda$ is a (negative) scaled phenomenological damping parameter; the overdot denotes differentiation with respect to $\tau$. The coefficients $A, B, C$ are likewise real and $O(1)$; expressions for $A, B, C$ in terms of $H, L, m$ and $n$ may be obtained from the results of [11], noting that equation (2.14c) of that paper is misprinted (in particular, a 1 should be added in the square bracket).

The SG experiment corresponds to $H=2.5 \mathrm{~cm}, L=6.17 \mathrm{~cm}$, $m=2$, and $n=3$; in the FS model, this gives $A=2.65$,
$B=-22.06$, and $C=-27.47$, values which are consistent with the values found in $[5,13]$, although scalings different from those in FS are used in these references. For this comparison we use the formulae in [5] omitting surface tension. With these coefficients the predicted bifurcation diagram for the SG experiment with $\omega>$ 0 is sketched in Fig. 1(a). Here the trivial state corresponds to the absence of surface waves, $\left(z_{1}, z_{2}\right)=(0,0)$, while the pure mode (PM) states correspond to equilibria of the form $\left(0, z_{2}\right)$ or $\left(z_{1}, 0\right)$, where $\left|z_{1}\right|=\left|z_{2}\right| \neq 0$, and the symmetric mixed mode (MM) states correspond to equilibria of the form $\left(z_{1}, \pm z_{1}\right), z_{1} \neq 0$; asymmetric general mode (GM) states in which both $z_{1}$ and $z_{2}$ are nonzero and $z_{1} \neq \pm z_{2}$ are not present for these parameter values. The bifurcation analysis of these fixed points is given in $[5,11,13]$.

Fig. 1(b) shows the experimentally obtained bifurcation diagram. The difference between the two diagrams is significant and remains unexplained. To shed light on the possible origin of this discrepancy it is helpful to examine the derivation of the amplitude equations for the $(2,3),(3,2)$ mode interaction from a more general point of view. To this end we consider a general mode interaction in a square domain subject to the assumption that the competing modes are related by symmetry and hence are in exact $1: 1$ temporal resonance, and that these modes have opposite parity under reflection in either midplane. The equations describing this mode interaction must be equivariant with respect to the group $\mathbf{D}_{4}$ generated by the actions

$\kappa_{1}:\left(z_{1}, z_{2}\right) \rightarrow\left(-z_{1}, z_{2}\right), \quad \gamma:\left(z_{1}, z_{2}\right) \rightarrow\left(z_{2}, z_{1}\right)$.

We further assume that the system is forced at twice the natural frequency of these modes and that this forcing is weak and hence breaks the time-translation symmetry only at linear order. Together these assumptions lead to amplitude equations whose third order truncation takes the form of Eqs. (2) albeit with complex coefficients. The resulting equations describe a parametrically forced Hopf bifurcation with $\mathbf{D}_{4}$ symmetry, and have been studied in $[14,15]$. The additional assumption that viscosity is small so that the cubic terms can be computed from inviscid theory, now leads to purely imaginary (i.e., energyconserving) cubic coefficients, and hence precisely the system (2). These results indicate that for the mode interaction studied in SG the form of Eqs. (2) remains unchanged both when viscosity and contact line motion are included in the derivation. This is despite the fact that both violate the assumed Neumann boundary conditions at the walls, and hence render the eigenfunctions nonsinusoidal. In fact the inclusion of viscosity has another effect as well-it is responsible for the presence of a streaming flow driven in viscous boundary layers at the surface, bottom and sides [1618]. This is a nonlinear effect of viscosity and is important because the resulting streaming flow in turn interacts with the surface oscillations responsible for its presence. Moreover, the generation of the streaming flow is a singular effect, and the streaming 

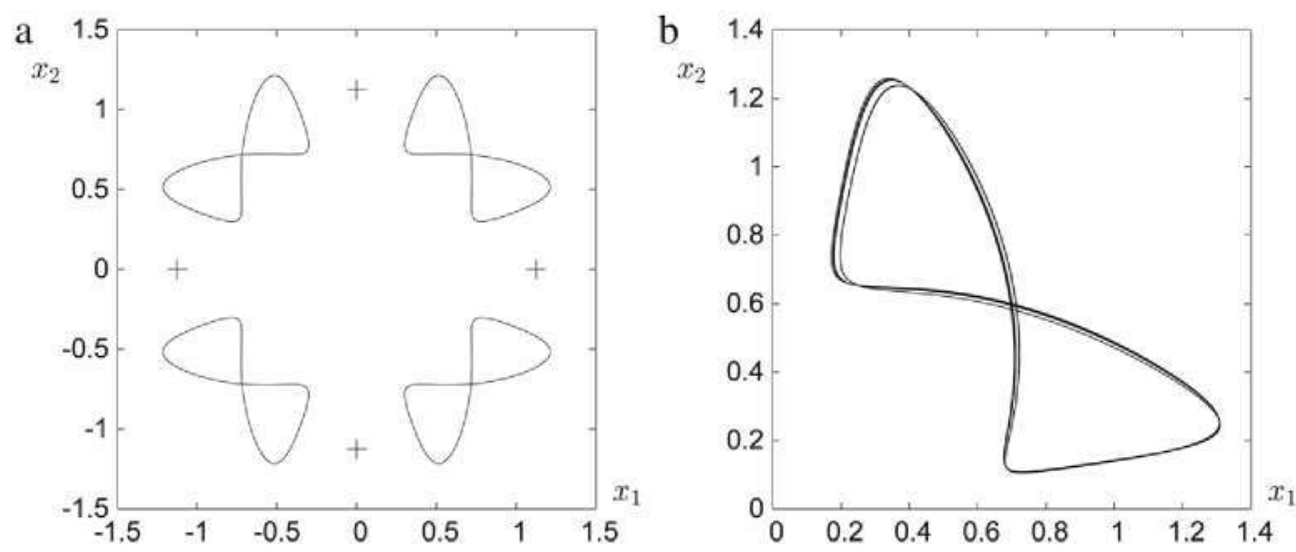

Fig. 2. (a) Four stable symmetry-related figure- 8 periodic orbits for $f=0.7$. PM states, which are saddles, are indicated by crosses. (b) Chaotic attractor for $f=0.46784$.

flow that is driven by the Reynolds stresses generated in the viscous boundary layers remains nonzero even in the limit of vanishing viscosity. Since the largest scales in the streaming flow are usually weakly damped the (unforced) streaming flow typically decays on a slow time scale unless maintained by the Reynolds stresses. It is well known that elimination of a weakly damped mode like the streaming flow via center-manifold reduction can substantially change the coefficients of the nonlinear terms in the normal form, although it does not change the form of the resulting equations [19]. Thus on time scales long compared with the viscous decay time of the unforced streaming flow the Faraday problem will still be described by Eqs. (2), albeit with (purely imaginary) coefficients $A, B, C$ that differ, perhaps substantially, from those computed on the basis of inviscid theory. The required centermanifold elimination of the streaming flow also reduces the range of validity of the resulting amplitude equations.

We conclude from this discussion that Eqs. (2) not only describe a particular idealization of the SG experiment but, in the case of unbroken square symmetry, the actual experiment itself. On the other hand the applicability to the SG experiment of the coefficient values computed by FS and listed above remains uncertain. It should be mentioned that these conclusions do not apply to all mode interactions-for some mode interactions the presence of Neumann boundary conditions and the associated hidden symmetries [20] is essential for the derivation of Eqs. (2), and in these cases the inclusion of viscosity and contact line motion will change the form of the amplitude equations.

In this paper we do not attempt to resolve these issues but focus instead on the properties of Eqs. (2) as derived in FS. Since most of the dynamical phenomena we identify are a consequence of the near-Hamiltonian nature of the system and its square geometry, we expect similar dynamics for other coefficient choices as well.

\section{Numerically observed periodic orbits}

For our numerical investigations we simplify Eqs. (2) by setting $\omega=1$ and $C=-1$. Provided these coefficients are nonzero and $\omega C<0$, this simplification is accomplished by the rescaling $\tau \rightarrow \tau /|\omega|,\left(z_{1}, z_{2}\right) \rightarrow \sqrt{-\omega / C}\left(z_{1}, z_{2}\right)$; if $\omega<0, z_{1}$ and $z_{2}$ have to be replaced by their complex conjugates. We match the remaining (rescaled) nonlinear coefficients with those obtained from the fluid equations for the SG experiment with $m=2, n=3$ by adopting the values $A=0.1$ and $B=-0.8$. Finally, we set $\lambda=-0.03$ and use $f$ as the control parameter. Physically, this corresponds to varying the forcing amplitude while keeping the system geometry, fluid properties, and forcing frequency (i.e., detuning) fixed.

Our results are typically presented in the form of projections on the $\left(x_{1}, x_{2}\right)$ plane, where $z_{j}=x_{j}+\mathrm{i} y_{j}, j=1,2$. For convenience, we also define the symmetry operation

$\rho=\kappa_{1} \gamma:\left(z_{1}, z_{2}\right) \rightarrow\left(-z_{2}, z_{1}\right)$,

which describes a rotation by $\pi / 2$.

\subsection{Figure-8 periodic orbits}

For $f=0.7$, we find four stable, symmetry-related periodic orbits as shown in Fig. 2(a); because of their shape in this projection, we refer to these as "figure- 8 " periodic orbits. Recall that periodic orbits for the model correspond to quasiperiodic surface waves in the physical system. These periodic orbits, with period $T=6.17$ at $f=0.7$, are symmetric under $\gamma$ (first and third quadrants in this projection) or $\rho^{-1} \gamma \rho$ (second and fourth quadrants in this projection). Using AUTO [21], we find that these orbits are stable between a saddle-node bifurcation at $f=0.7771864$ and a symmetry-breaking bifurcation at $f=0.4862516$. This symmetry-breaking bifurcation produces a branch of stable, asymmetric figure- 8 periodic orbits. These remain stable as $f$ decreases until a period-doubling bifurcation at $f=$ 0.4700954 ; this is the beginning of a period-doubling cascade which gives rise to symmetry-related chaotic attractors, one of which is shown in Fig. 2(b) at $f=0.46784$. We note that the basin of attraction of this chaotic attractor is quite small. Furthermore, these results illustrate the fact that a symmetric periodic orbit, such as the original figure- 8 periodic orbits, must first undergo a symmetry-breaking bifurcation before a period-doubling cascade can occur [22].

The branch of symmetric figure- 8 periodic orbits of the type shown in Fig. 2(a) can be numerically continued in both directions using AUTO [21], revealing two distinct global bifurcations wherein the orbits collide with pairs of symmetry-related PM equilibria. One such global bifurcation, hereafter referred to as $\mathrm{GB}_{1}$, occurs at $f=0.04496453$, while the other, $\mathrm{GB}_{2}$, occurs at $f=0.04476048$. The $\mathrm{PM}$ eigenvalues near $\mathrm{GB}_{1}$ and $\mathrm{GB}_{2}$ are, approximately, 0.331 and -0.391 within the pure mode subspace, and 0.81 and -0.87 transverse to this subspace. The shapes of the heteroclinic connections between $\mathrm{PM}$ at $\mathrm{GB}_{1}$ and $\mathrm{GB}_{2}$ are shown in Fig. 3 .

The branch of asymmetric figure- 8 periodic orbits that arises in the symmetry-breaking bifurcation at $f=0.4862516$ can be numerically followed to a global bifurcation at $f=0.04493764$. At this bifurcation, the trajectories involve visits to (or near) PM solutions as shown in Fig. 4(a). This global bifurcation occurs at a value of $f$ that is very close to the values at which $G_{1}$ and $\mathrm{GB}_{2}$ occur, Although this bifurcation is most likely homoclinic (otherwise it would be of codimension two), the associated orbit resembles a composition of heteroclinic connections taken from those generated at $\mathrm{GB}_{1}$ and $\mathrm{GB}_{2}$; this is shown clearly in 

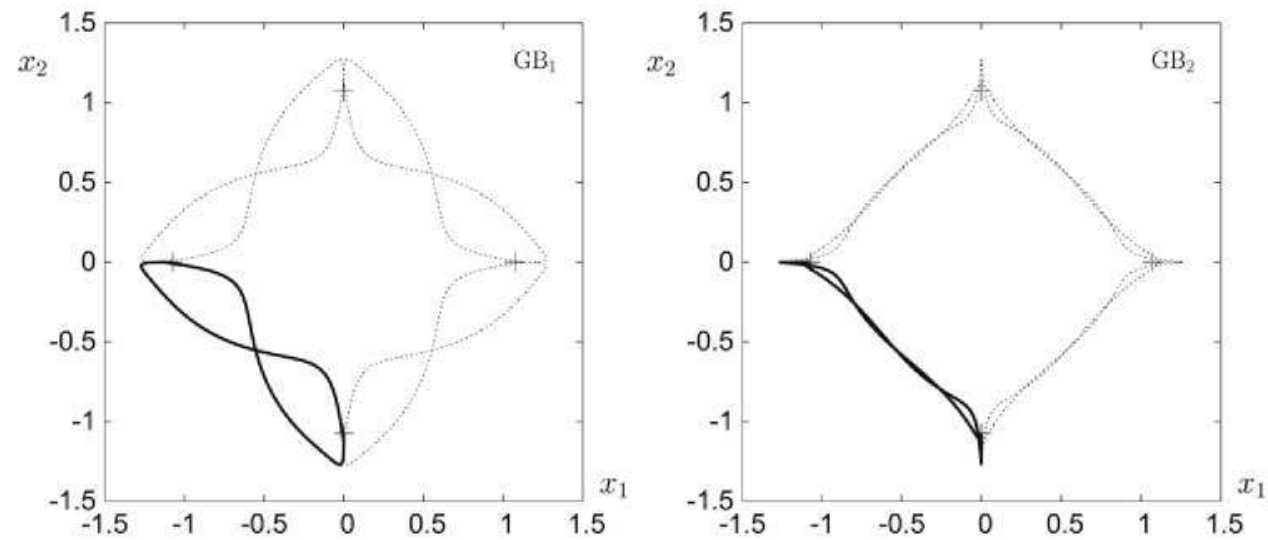

Fig. 3. Connections between PM equilibria (marked with a cross) at the global bifurcations $\mathrm{GB}_{1}$ (left), with $f=0.04496453$, and $\mathrm{GB}_{2}$ (right), with $f=0.04476048$. Connections associated with the specific periodic orbit in quadrant III of Fig. 2(a) are shown in bold.
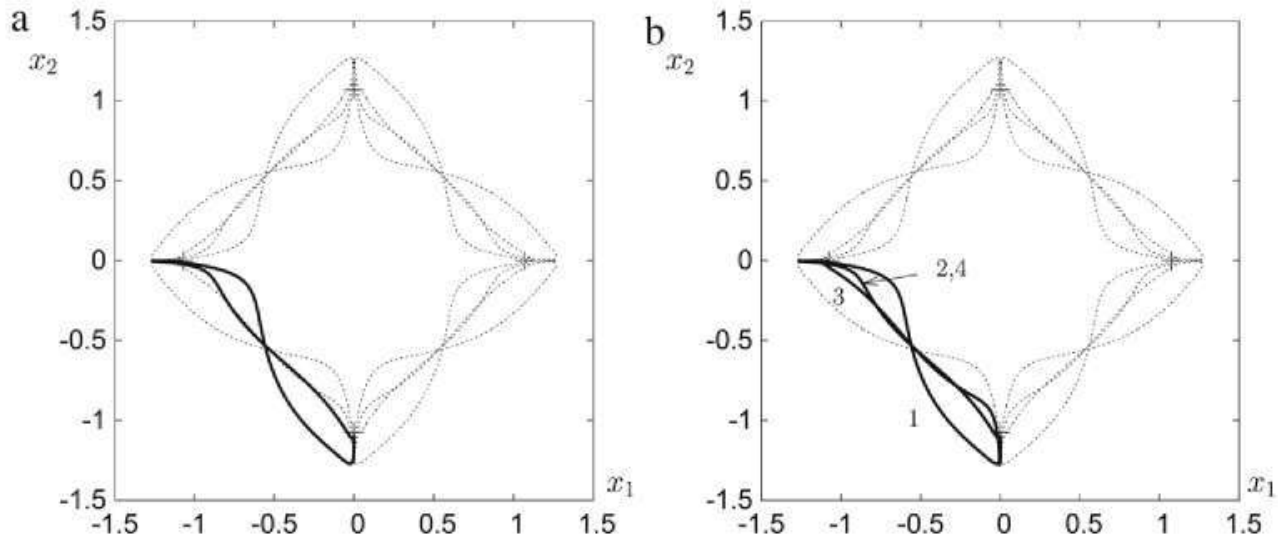

Fig. 4. (a) Trajectory (solid line) at the global bifurcation at $f=0.04493764$, found by following an asymmetric figure- 8 periodic orbit. (b) Trajectory (solid line) at the global bifurcation at $f=0.04493380$, found by following the periodic orbit which arises from the period-doubling bifurcation of an asymmetric figure- 8 periodic orbit at $f=0.4700954$. The dashed lines show connections which exist at $\mathrm{GB}_{1}$ and $\mathrm{GB}_{2}$, which occur at slightly different values of $f$. In this and subsequent figures the numbers indicate the sequence of approximate heteroclinic connections traced out by the trajectory; where two numbers are given, approximately the same path is traced out twice.

Fig. 4, which includes the connections present at $\mathrm{GB}_{1}$ and $\mathrm{GB}_{2}$ as dashed lines. Likewise, the branch arising from the perioddoubling bifurcation on the asymmetric figure-8 periodic orbit branch at $f=0.4700954$ can be followed to a (homoclinic) global bifurcation at $f=0.04493380$ that resembles another combination of heteroclinic connections between PM solutions, as shown in Fig. 4(b).

The bifurcation diagram in Fig. 5 summarizes the above results for figure- 8 orbits, placing them alongside corresponding results for the star periodic orbits described below in Section 3,2.

We remark that the figure- 8 periodic orbits resemble the periodic orbit shown in Figure 16 of [3], except that the experimentally observed solution appears to be symmetric under $\kappa_{1}$ rather than $\gamma$. From this we conjecture that the periodic solution in [3] might be associated with a heteroclinic bifurcation involving MM rather than PM solutions. In this connection we recall that in the experimental system MM solutions are in fact unstable for sufficiently large $f$, as shown in Fig. 1(b).

\subsection{Star periodic orbits}

Another type of stable periodic orbit, shown in Fig. 6(a), can also be found at $f=0.7$; because of its shape in this projection, we refer to this as a "star" periodic orbit. This periodic orbit, with period $T=11.97$ for $f=0.7$, is symmetric under $\rho$. The action of $\kappa_{1}$ or $\gamma$ generates a symmetry-related star periodic orbit, which rotates in the opposite sense. Using numerical continuation, we establish the stability of star periodic orbits for $0.571786<f<0.9955955$ and, on another part of the branch, for $0.8225886<f<0.9197870$; see Fig. 5.

The symmetric star periodic orbit loses stability at $f=$ 0.571786 through a symmetry-breaking bifurcation, giving rise to a stable periodic orbit shown for $f=0.54$ in Fig. 6(b). This orbit is symmetric under $\rho^{2}$ (rotation by $\pi$ ). As $f$ decreases, this branch itself undergoes a symmetry-breaking bifurcation that produces a stable asymmetric periodic orbit, shown in Fig. 6(c) for $f=0.515$. As $f$ decreases still further, there is a period-doubling cascade to chaos; a chaotic attractor at $f=0.51255$ is shown in Fig. 6(d). We note that the basin of attraction of this chaotic attractor is quite small. The branch containing the star periodic orbit shown in Fig. 6(a) can be numerically continued in both directions using AUTO, leading to the same two global bifurcations, $G_{1}$ and $G_{2}$, defined above. The connections between PM equilibria obtained in this fashion are shown in Fig. 7, which should be compared with Fig. 3.

The $\rho^{2}$-symmetric periodic orbit shown in Fig. $6($ b) can be continued numerically until a global bifurcation at $f=$ 0.04493765 , where the trajectory makes visits to (or very near) PM solutions as shown in Fig. 8(a). As in the case of the asymmetric figure- 8 orbits, this global bifurcation occurs at a value of $f$ that is very close to the values for $G_{1}$ and $G_{2}$. Fig. 8(a), which includes the connections present at $\mathrm{GB}_{1}$ and $\mathrm{GB}_{2}$ (dashed lines), indicates that the heteroclinic connections along this orbit, whether exact or approximate, again resemble a combination of the connections generated at $\mathrm{GB}_{1}$ and $\mathrm{GB}_{2}$. Similarly, the asymmetric periodic orbit shown in Fig. 6(c) can be followed to a global bifurcation at 

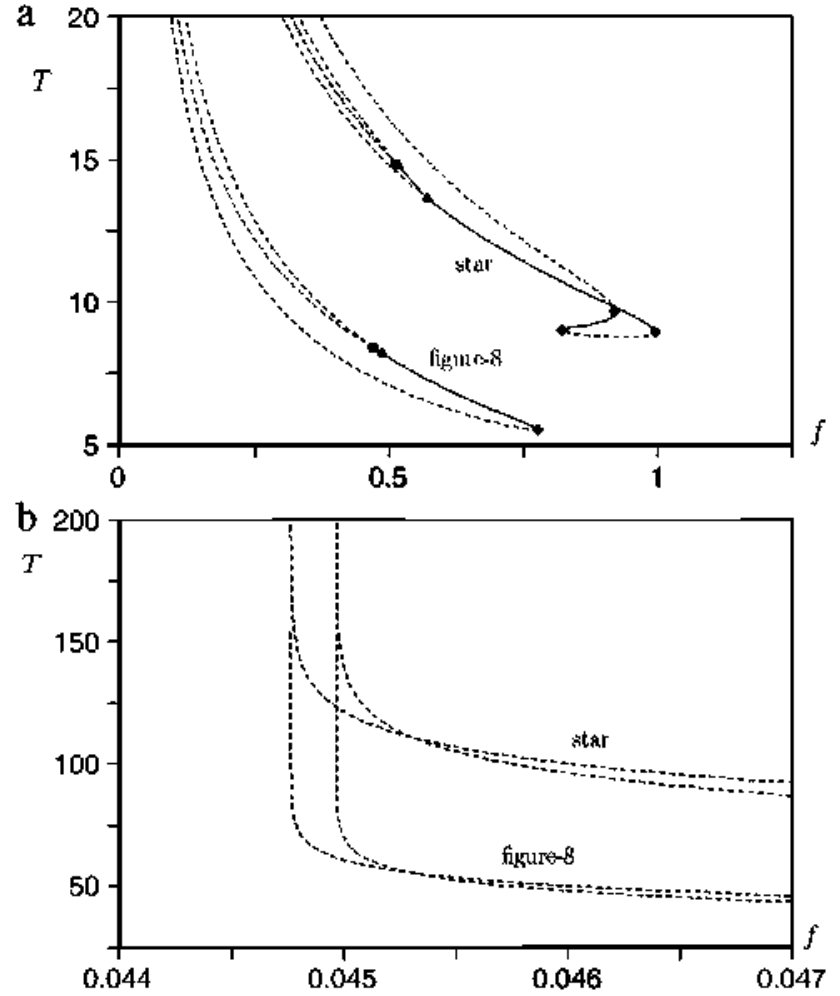

Fig. 5. (a) Bifurcation diagram for the figure-8 periodic orbits of Fig. 2(a) and the star periodic orbits of Fig. $6($ a), as well as related periodic orbits described in the text. For clarity, branches arising from period-doubling bifurcations are not shown. (b) Enlargement showing the divergence of the period $T$ of the figure- 8 and star periodic orbits as the global bifurcations $G B_{1}$ and $C B_{2}$ are approached. In this and other bifurcation diagrams, diamonds, squares, and triangles indicate saddle-node. period-doubling, and symmetry-breaking pitchfork bifurcations, respectively. Furt hermore, solid (dashed) lines represent stable (unstable) solutions.

$f=0.04496433$, producing connections among the PM shown in Fig. 8(b); this, too, resembles a composite of the connections realized at $\mathrm{GB}_{1}$ and $\mathrm{GB}_{2}$.

At this point it is appropriate to mention that for these values of the coefficients a variety of other stable periodic orbits can be found as well, as described in the Appendix. We find that some of these are also related to the heteroclinic connections identified above, since they terminate, when followed in $f$, in global bifurcations involving connections resembling those at $\mathrm{GB}_{1}$ and $\mathrm{GB}_{2}$. Others exist on isolas.

It is of interest that dynamics similar to those described thus far, including versions of the figure- 8 and star periodic orbits, are also present in the work of Abreu et al. [23] on a $\mathbf{D}_{4}$-symmetric system in $\mathbb{R}^{3}$ describing steady-state/steady-state mode interaction. Although these authors did not attempt to locate global bifurcations, they observed and investigated chaotic attractors like those shown in Fig. 2(b) and 6(d); chaotic attractors in their system seem to be considerably more robust than in the system (2).

\subsection{Dependence of dynamics on damping}

Qualitatively similar behavior is observed when other parameters are also allowed to vary. For example, with $\lambda=-0.02$ (less damping) and the remaining parameters as above, we find analogues of $\mathrm{GB}_{1}$ and $\mathrm{GB}_{2}$ at $f=0.03183159$ and $f=0.03235626$. For $\lambda=-0.01$ and the remaining parameters unchanged, we find analogues of $\mathrm{GB}_{1}$ and $\mathrm{GB}_{2}$ at $f=0.01818218$ and $f=0.01899046$.
On the other hand, continuing the figure- 8 periodic orbits with respect to for $\lambda=-0.05$ or $\lambda=-0.1$ reveals that these solutions no longer terminate in global bifurcations but, instead, lie on isolas, as illustrated in Fig. 9. This suggests that the two global bifurcations come together for larger damping and "pinch off" to create the isola (see Section 5),

\section{Hamiltonian limit}

Most of the periodic orbits described in Section 3 either originate directly in heteroclinic bifurcations or are evidently associated with them. These heteroclinic connections are, in turn, associated with the Hamiltonian limit of Eqs. (2), as we now describe, cf. $[24,25]$. The analysis of this limiting case employs standard techniques but these are applied here to the case of persistent heteroclinic connections (a consequence of the unbroken $D_{4}$ symmetry), a situation that has not, to the best of our knowledge, been fully considered.

Eqs. (2) have a Hamiltonian structure when damping is neglected $(\lambda=0)$, and can be obtained from the Hamiltonian

$$
\begin{aligned}
H_{z}= & \omega\left(\left|z_{1}\right|^{2}+\left|z_{2}\right|^{2}\right)+\frac{\mathrm{i} f}{2}\left(z_{1}^{2}-\bar{z}_{1}^{2}+z_{2}^{2}-\bar{z}_{2}^{2}\right)-B\left|z_{1}\right|^{2}\left|z_{2}\right|^{2} \\
& +\frac{A+B}{2}\left(\left|z_{1}\right|^{2}+\left|z_{2}\right|^{2}\right)^{2}+\frac{C}{2}\left(z_{1}^{2} \bar{z}_{2}^{2}+\bar{z}_{1}^{2} z_{2}^{2}\right)
\end{aligned}
$$

via

$\dot{z}_{\mathrm{j}}=\mathrm{i} \frac{\partial H_{z}}{\partial \bar{z}_{j}}, \quad \dot{\bar{z}}_{j}=-\mathrm{i} \frac{\partial H_{z}}{\partial z_{j}}, \quad j=1,2$.

If we further set $f=0$ then Eqs. (2) are integrable, and the energy $E=\left|z_{1}\right|^{2}+\left|z_{2}\right|^{2}$ constitutes a second invariant (in addition to $\mathrm{H}_{2}$ ). This integrable system boasts an infinite number of heteroclinic trajectories. Before describing these, however, it is convenient to introduce another set of coordinates with the transformation (see, e.g. [25])

$z_{j}=\sqrt{2 J_{j}} \mathrm{e}^{i \theta_{j}}, \quad j=1,2, I_{j}>0$,

to obtain the amplitude-phase system

$i_{1}=2 \lambda I_{1}+2 I_{1} \cos \left(2 \theta_{1}\right)+4 C I_{1} I_{2} \sin \left(2 \theta_{1}-2 \theta_{2}\right)$,

$\dot{\theta}_{1}=\omega-f \sin \left(2 \theta_{1}\right)+2 A\left(I_{1}+I_{2}\right)+2 B I_{1}+2 I_{2} \cos \left(2 \theta_{1}-2 \theta_{2}\right)$,

$j_{2}=2 \lambda I_{2}+2 f I_{2} \cos \left(2 \theta_{2}\right)-4 C I_{1} I_{2} \sin \left(2 \theta_{1}-2 \theta_{2}\right)$.

$\dot{\theta}_{2}=\omega-f \sin \left(2 \theta_{2}\right)+2 A\left(I_{1}+I_{2}\right)+2 B I_{2}+2 C I_{1} \cos \left(2 \theta_{1}-2 \theta_{2}\right)$.

The system (6) is, of course, also Hamiltonian when $\lambda=0$ and can be obtained from

$$
\begin{aligned}
H_{i \theta}= & \frac{1}{2} H_{z}=\omega\left(I_{1}+I_{2}\right)-f\left[I_{1} \sin \left(2 \theta_{1}\right)+I_{2} \sin \left(2 \theta_{2}\right)\right] \\
& +(A+B)\left(I_{1}+I_{2}\right)^{2}+2 I_{1} I_{2}\left[C \cos \left(2 \theta_{1}-2 \theta_{2}\right)-B\right]
\end{aligned}
$$

via

$\dot{\theta}_{\mathrm{j}}=\frac{\partial H_{j \theta}}{\partial J_{j}}, \quad \dot{\mathrm{j}}_{\mathrm{j}}=-\frac{\partial H_{i \theta}}{\partial \theta_{j}}, \quad j=1,2$.

Finally, for certain purposes it is useful to consider a third set of coordinates defined (see [26]) by

$I=I_{1}+I_{2}, \quad \theta=\theta_{1}, \quad x=\sqrt{2 I_{2}} \cos \left(\theta_{1}-\theta_{2}\right)$,

$y=\sqrt{2 I_{2}} \sin \left(\theta_{1}-\theta_{2}\right)$,

and leading to the system

$\dot{x}=\lambda x+f[x \cos (2 \theta)+2 y \sin (2 \theta)]+2(B+C)\left(y^{2}-I\right) y+2 B x^{2} y$,

$\dot{y}=\lambda y-f y \cos (2 \theta)+2(C-B)\left(x^{2}-I\right) x-2 B y^{2} x$,

$\dot{I}=2 \lambda I+2 f\left[\left(I-y^{2}\right) \cos (2 \theta)+x y \sin (2 \theta)\right]$,

$\dot{\theta}=\omega-f \sin (2 \theta)+2(A+B) I+(C-B) x^{2}-(B+C) y^{2}$. 

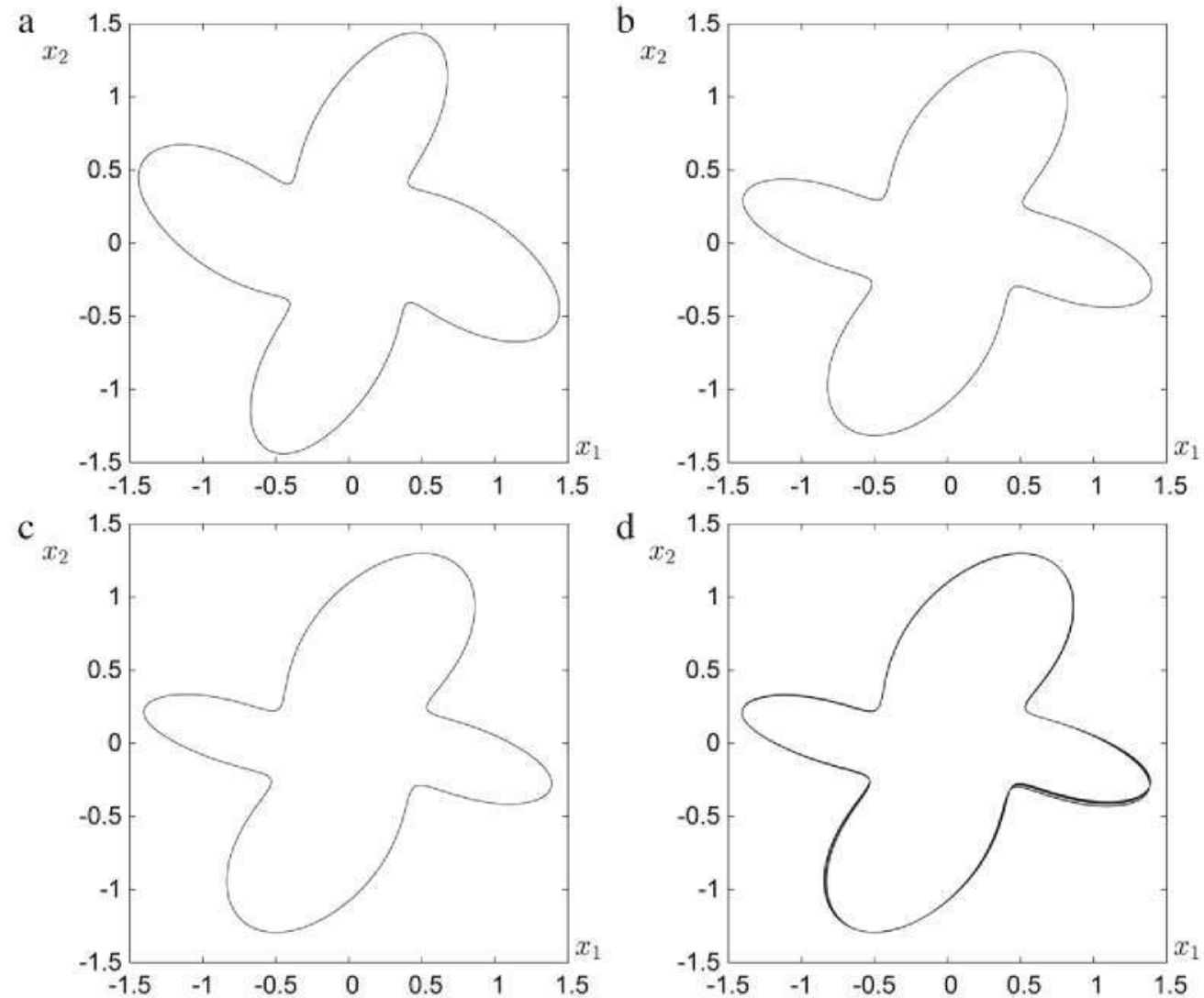

Fig. 6. (a) Stable star periodic orbits for (a) $f=0.7$, (b) $f=0.54$, and (c) $f=0.515$. (d) Chaotic attractor for $f=0.51255$.
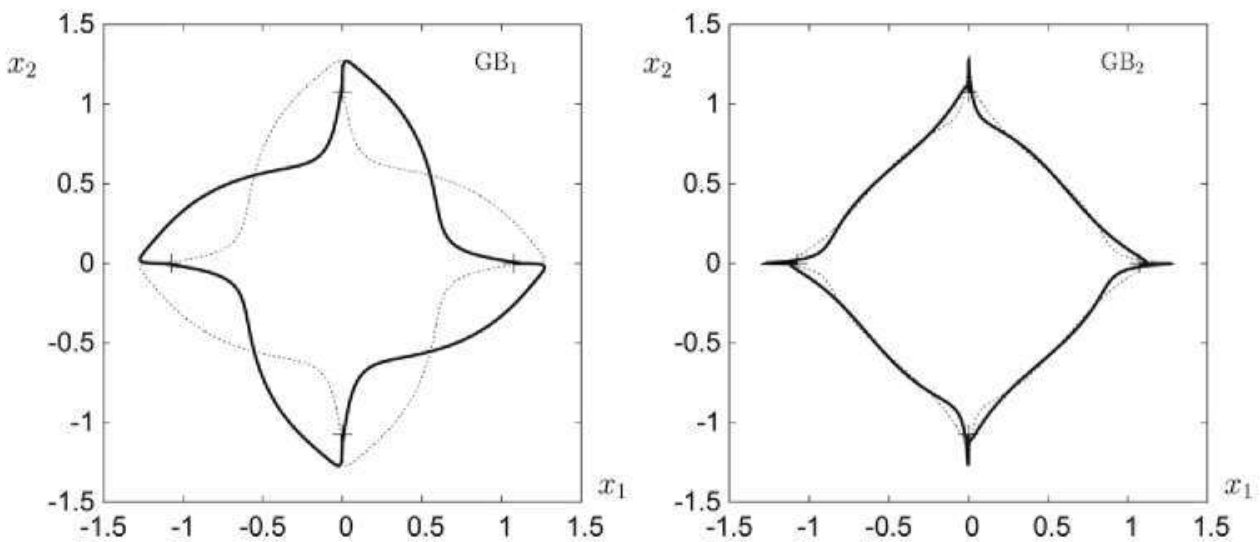

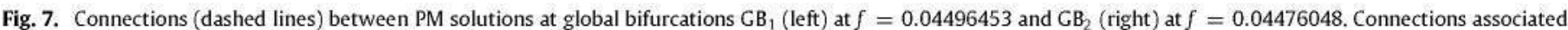
with a single star periodic orbit from Fig. 6(a) are shown in bold.

Note that although we $\operatorname{set} \theta=\theta_{1}$ and used $I_{2}$ in the definition of $x$ and $y$, the symmetry $\gamma$ implies that Eqs. (10) would have resulted from the complementary choice as well.

We now describe the phase-space structure of the twodimensional pure mode subspaces $\mathscr{P}_{1}\left(z_{2}=0\right)$ and $\mathcal{P}_{2}\left(z_{1}=0\right)$ obtained, e.g., from setting $x=y=0$ in Eqs. (10) to yield the two-dimensional system

$\dot{I}=2 \lambda I+2 f I \cos (2 \theta)$,

$\dot{\theta}=\omega-f \sin (2 \theta)+2(A+B) I$.

The dynamics of such systems were analyzed in [27]. We show in Fig. 10 the phase-space structure in three relevant cases: (a) $\lambda=f=0$, (b) $\lambda=0, f>0$, and (c) $\lambda<0, f>0$. In the integrable
Hamiltonian limit (a), the pure mode dynamics are particularly simple since $i=0$. There is then an isolated fixed point at the origin and, provided $-\omega /(2(A+B)) \equiv \omega r_{0}>0$ as in Section 3 , a circle of fixed points at $I=\omega r_{0}$, while the remaining trajectories are all circular periodic orbits; see Fig. 10(a). When the forcing $f$ is added, as in Fig. 10(b), the circle of fixed points collapses, leaving two types of pure mode equilibria: saddles with amplitude $I=(\omega-f) r_{0}$ at $\theta=\pi / 4,5 \pi / 4$ and centers with amplitude $I=(\omega+f) r_{0}$ at $\theta=-\pi / 4,-5 \pi / 4$. When damping $0<-\lambda<f$ is included as well, the saddles move to $I=\left(\omega-\sqrt{f^{2}-\lambda^{2}}\right) r_{0}$ and $\theta=\tilde{\theta}, \pi+\tilde{\theta}$ where $\tilde{\theta}=\cos ^{-1}(-\lambda / f) / 2$, while the centers become sinks at $I=\left(\omega+\sqrt{f^{2}-\lambda^{2}}\right) r_{0}$ and $\theta=-\tilde{\theta}, \pi-\tilde{\theta}$. 

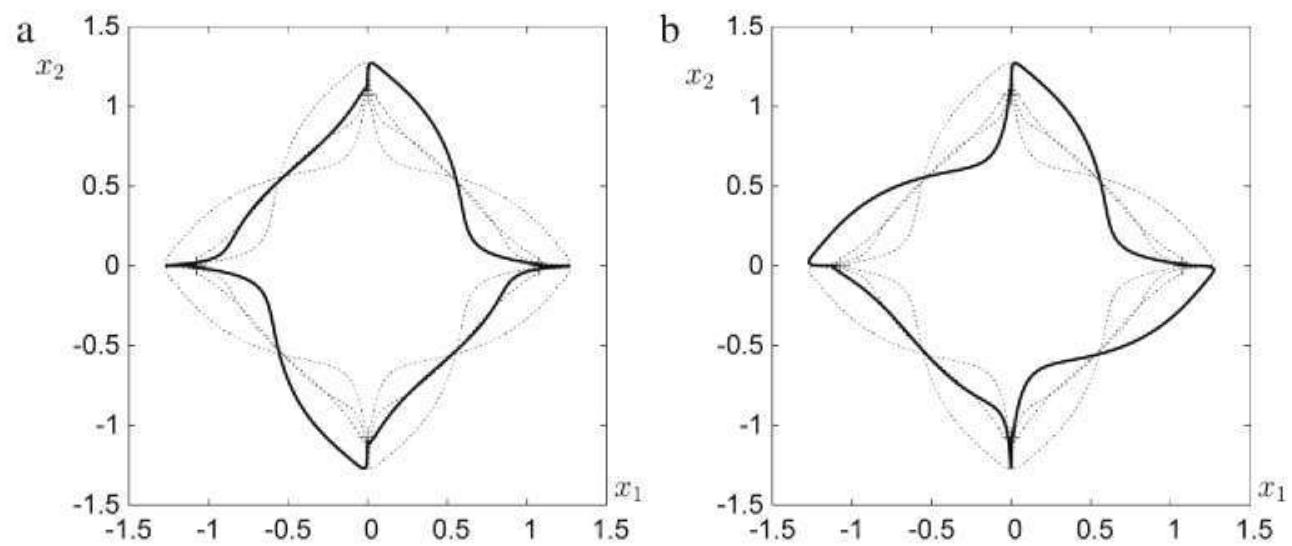

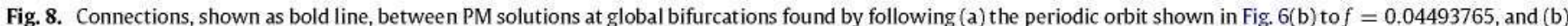
the periodic orbit shown in Fig. $6(\mathrm{c})$ to $f=0.04496433$. The dashed lines show connections which exist at $\mathrm{GB}_{1}$ and $\mathrm{GB}_{2}$.
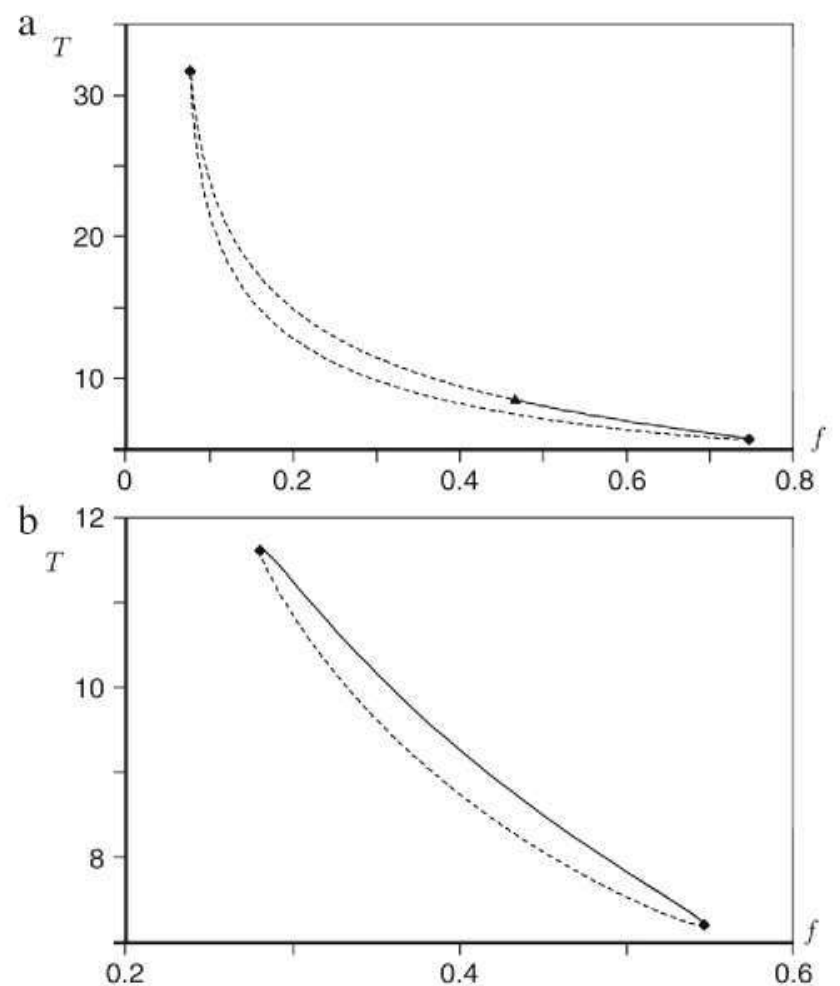

Fig. 9. Bifurcation diagrams for figure- 8 periodic orbits with (a) $\lambda=-0.05$ and (b) $\lambda=-0.1$. The periodic orbits lie on isolas when followed with respect to $f$, rather than terminating in global bifurcations.
While Fig. 10(b) shows the presence when $\lambda=0, f>$ 0 of heteroclinic connections between pure modes of the same type (e.g., within $\mathscr{P}_{1}$, related by $\kappa_{1}$ ) the heteroclinic behavior discovered in Section 3 involves connections between $\mathscr{P}_{1}$ and $\mathscr{P}_{2}$. This type of heteroclinic connection can be most easily seen with the system (10) in the integrable Hamiltonian limit. In this case $(\lambda=f=0$ ) the dynamics of $x$ and $y$ can be examined separately since $I$ is conserved and, hence, may be treated as a parameter, while $\theta$ decouples entirely. Thus we consider the equations

$\dot{x}=-2(B+C) I y+2 B x^{2} y+2(B+C) y^{3}$,

$\dot{y}=2(B-C) I x-2 B y^{2} x+2(C-B) x^{3}$,

which can be derived from the reduced Hamiltonian

$H_{x y}=\frac{1}{2}(B-C)\left(x^{2}+y^{2}-2 I\right)\left(x^{2}+\frac{B+C}{B-C} y^{2}\right)$

via

$\dot{x}=\frac{\partial H_{x y}}{\partial y}, \quad \dot{y}=-\frac{\partial H_{x y}}{\partial x}$.

This two-dimensional system exhibits three types of fixed points: a "pure mode" (PM) with $x=y=0$, corresponding in the full system (6) to the entire subspace $\mathscr{P}_{1}$, mixed modes (MM) with $x^{2}=I, y=0$ or $y^{2}=I, x=0$, meaning that $I_{1}=I_{2}$ in system (6), and $\mathrm{PM} \in \mathscr{P}_{2}$ with $x^{2}=(B+C) I / C, y^{2}=(C-B) I / C$, implying that $I_{1}=0$ in Eqs. (6). This structure is depicted in Fig. 11.

The heteroclinic trajectories, which are sketched in Fig. 11, can be obtained explicitly by noting that $H_{x y}=0$ in both pure mode subspaces and, hence, must also vanish along connecting orbits, which in this case implies that

$(B+C) y^{2}=(C-B) x^{2}$.
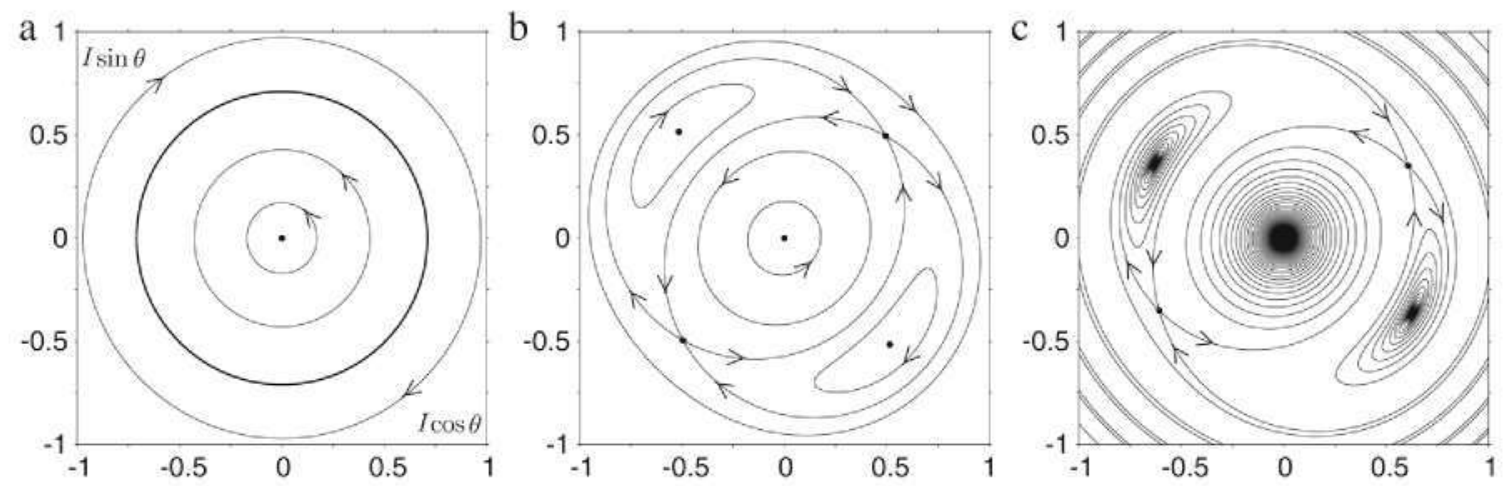

Fig. 10. Dynamics in the pure mode subspace with (a) $\lambda=f=0$, (b) $\lambda=0, f>0$, and (c) $\lambda<0, f>0$. The remaining parameters are as in Section 3 . 


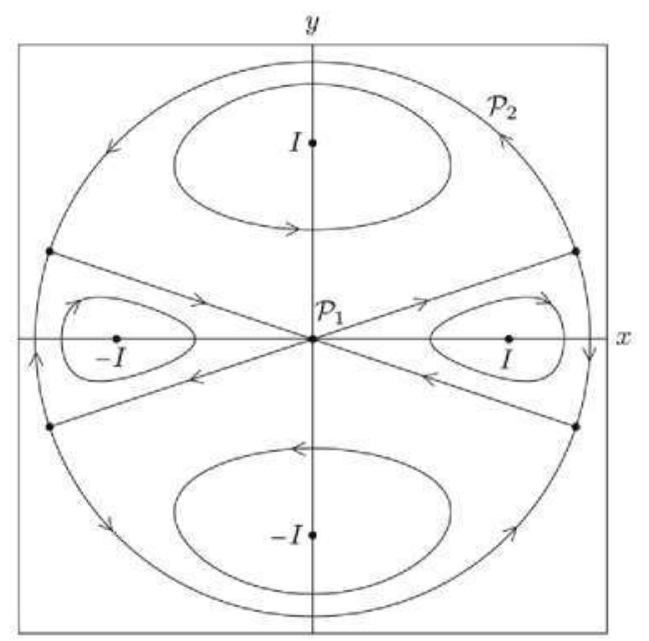

Fig. 11. Dynamics transverse to the pure mode subspace $\mathscr{P}_{1}$, described by Eqs. (12). Note the heteroclinic cycles connecting this subspace, located at the origin, to the other pure mode subspace $\mathscr{P}_{2}$, represented here by the circle $x^{2}+y^{2}=2 I$.

This relation may be substituted into Eq. (12a) and the result integrated to yield

$x^{2}(t)=\frac{(B+C) I}{C\left(1+\mathrm{e}^{ \pm 4 I \sqrt{C^{2}-B^{2}} t}\right)}$.

Here we have taken the initial condition as $x^{2}(0)=(B+C) I /(2 C)$. In the "-" case, Eq. (16) describes, for $-\infty<t<\infty$, a trajectory from $\mathscr{P}_{1}$ (the origin) to $\mathscr{P}_{2}\left(x^{2}+y^{2}=2 I\right)$. Once in $\mathscr{P}_{2}$, trajectories "rotate" (moving along one of the four arcs in Fig. 11) toward one of the unstable transverse directions, signified by the fixed points in the second and fourth quadrants, and then, finally, back to $\mathscr{P}_{1}$ along the trajectory described by Eq. (16) in the "+" case.

We emphasize that these heteroclinic connections constitute a two-parameter family in the full system (10), parametrized by $I$ and $\theta$. In other words, each of the circular periodic orbits of Fig. 10 (a) in the $\mathscr{P}_{1}$ subspace is connected to its counterpart in the $\mathscr{P}_{2}$ subspace, and, considered as a whole, both $\mathscr{P}_{1}$ and $\mathscr{P}_{2}$ are normally hyperbolic invariant manifolds (see, e.g., [28]) with coincident three-dimensional stable and unstable manifolds, i.e., $\mathrm{W}^{\mathrm{u}}\left(\mathscr{P}_{1}\right)=\mathrm{W}^{\mathrm{s}}\left(\mathscr{P}_{2}\right)$ and $\mathrm{W}^{\mathrm{u}}\left(\mathscr{P}_{2}\right)=\mathrm{W}^{\mathrm{s}}\left(\mathscr{P}_{1}\right)$.

In the undamped, unforced version of system (6), which treats both pure mode subspaces symmetrically, these same heteroclinic orbits are expressed as

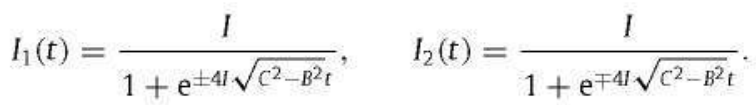

With the upper signs Eq. (17) describes, for $-\infty<t<\infty$, a heteroclinic orbit from $\mathscr{P}_{1}$ to $\mathscr{P}_{2}$, and vice versa for the lower signs. On these heteroclinic orbits the phases $\theta_{1}$ and $\theta_{2}$ evolve uniformly and at the same rate. This result follows from Eqs. (9) and (15), which imply that $C \cos \left(2\left(\theta_{1}-\theta_{2}\right)\right)-B=0$ on heteroclinic trajectories. As a result Eqs. (6b) and (6d) can be readily integrated, giving

$\theta_{j}(t)=\theta_{j}(0)+[\omega+2(A+B) I] t, \quad j=1,2$.

\section{Persistence of heteroclinic cycles in the perturbed system}

In this section we provide numerical evidence that certain of the heteroclinic connections present in the Hamiltonian limit persist when small dissipation is added, and conjecture that the number of such connections increases without bound as $\lambda$ decreases to zero.

The codimension-one heteroclinic orbits of interest are all unstable because they are contained in a two-dimensional unstable manifold (associated with two real and distinct positive eigenvalues). Hence, they are difficult to locate numerically. In Section 3 we were able to locate $G_{1}$ and $G_{2}$, as well as several additional global bifurcations, by following initially stable periodic orbits to very high period, which is indicative of a global bifurcation. Such high-period periodic orbits may also be continued in two parameters using AUTO (keeping the period fixed) to give a good indication of the heteroclinic bifurcation set. This has been done in Fig. 12(a), beginning from a figure- 8 periodic orbit of period $T=2000$ approximating $\mathrm{GB}_{1}$, and allowing both $f$ and $\lambda$ to vary.

Fig. 12 shows that, as $f$ increases, the heteroclinic bifurcation $\mathrm{GB}_{1}$ approaches a limit point (LP) near $f=0.057176, \lambda=$ -0.039963 . The new (lower) branch that emerges from this LP approximates $\mathrm{GB}_{2}$. For $f<0.057176$ these two branches remain close, first separating slightly, but then drawing nearer again as $f$ decreases. Nonetheless, they do not cross and appear to terminate at distinct points on the $f$ axis: $f=0.00191$ for $\mathrm{GB}_{1}$ and $f=0.00139$ for $\mathrm{GB}_{2}$. A close-up showing these two termination points is provided in Fig. 12(b). The proximity of these termination points to $f=\lambda=0$ supports the argument of Section 4 that the underlying source of these heteroclinic cycles is the integrable Hamiltonian limit with its two-parameter family of heteroclinic orbits. By the same token, the failure of these two branches, $\mathrm{GB}_{1}$ and $\mathrm{GB}_{2}$, to terminate exactly at $f=0$ is a strong indication that they are not unique and that other such
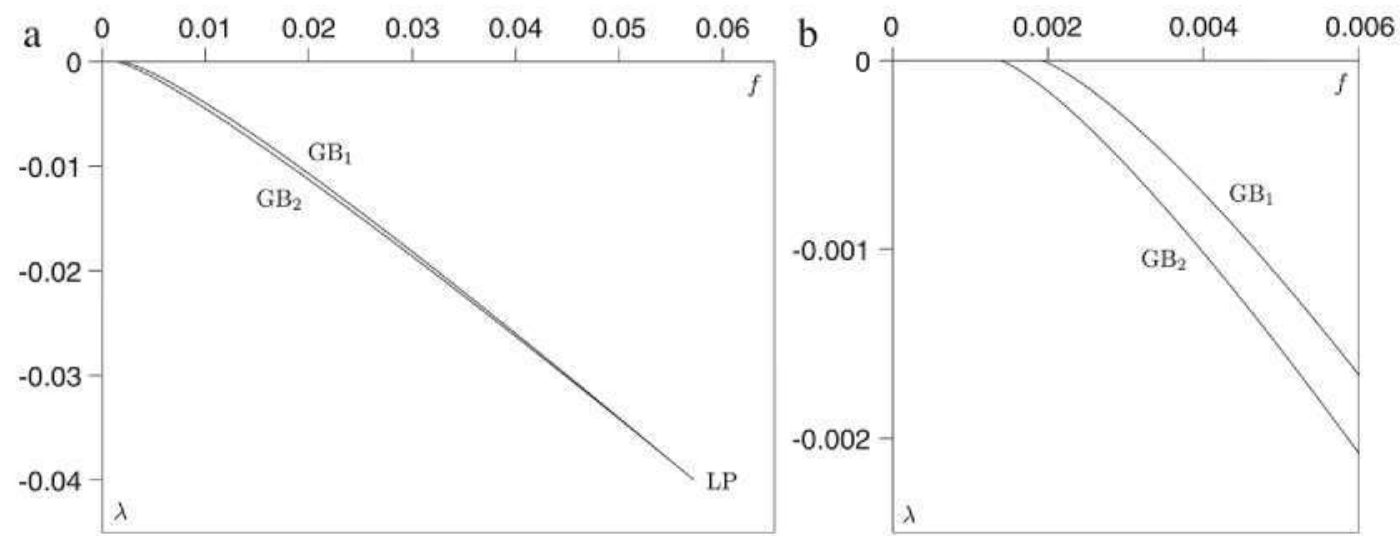

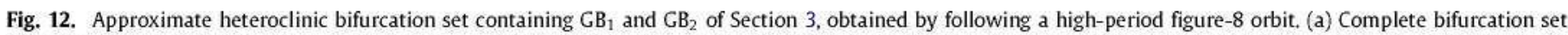

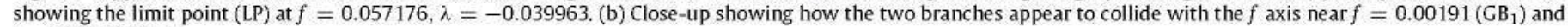
$f=0.00139\left(\mathrm{~GB}_{2}\right)$. 
branches emerge from different points along the $f$ axis, where the system (2) is Hamiltonian but not integrable. Presumably some of these are associated with the other global bifurcations described in the Appendix.

Unfortunately, it is not easy to search for heteroclinic cycles in system (2). Not only are they unstable, as mentioned earlier, but they are resistant to standard shooting methods. The main difficulty is that trajectories approximating heteroclinic connections must spend very long times in the vicinity of invariant subspaces ( $\mathscr{P}_{1}$ and $\mathscr{P}_{2}$ ) having unstable transverse directions, and this makes them extremely sensitive to numerical errors. In our case the problem is exacerbated by the fact that the transverse unstable direction is the most unstable direction (for $\lambda=-0.03$, near $G_{1}$, the unstable transverse eigenvalue of the saddle is 0.81 while the eigenvalue describing unstable pure mode perturbations is 0.331). To approximate orbits, for example, that leave the saddle PM and spiral many times toward the stable sink within $\mathscr{P}_{1}$ (see Fig. $10\left(\right.$ c)) before finally veering away toward $\mathscr{P}_{2}$, it is necessary to apply a modified integration procedure that effectively "turns off" the transverse dynamics while the orbit remains close to $\mathscr{P}_{1}$ (see, e.g., [29]). We utilize such a procedure here, and a suitable Poincaré section, to visualize the intersection of $W^{u}\left(P_{1}\right)$ and $W^{s}\left(P_{2}\right)$, the unstable and stable manifolds of the relevant saddle fixed points in $\mathscr{P}_{1}$ and $\mathscr{P}_{2}$, respectively.

Fig. 13 shows the results of a numerical procedure wherein we first integrate within the invariant subspace $\mathscr{P}_{1}$, using Eqs. (11) and starting near the saddle fixed point $\mathrm{PM}_{1}$. This provides an approximation of $W^{u}\left(P_{1}\right) \cap \mathcal{P}_{1}$. We then step along this trajectory, and at each step integrate forward again in time with Eqs. (6) while adding an initial transverse perturbation $I_{2}=\varepsilon$ (we take $\varepsilon=10^{-5}$ ). These integrations are stopped when the condition $I_{1}=I_{2}$ is satisfied. The same thing is done in reverse time for $\mathrm{PM}_{2} \in \mathcal{P}_{2}$. In this manner we obtain approximate representations of the intersections of $\mathrm{W}^{\mathrm{u}}\left(\mathrm{PM}_{1}\right)$ and $\mathrm{W}^{\mathrm{s}}\left(\mathrm{PM}_{2}\right)$ with the Poincare section $I_{1}=I_{2}$.

In Fig. 13(a) we show these intersections for $f=0.04496453$ and $\lambda=-0.03$, the location of $\mathrm{GB}_{1}$. In addition to the intersection of $\mathrm{W}^{\mathrm{u}}\left(\mathrm{PM}_{1}\right)$ and $\mathrm{W}^{\mathrm{s}}\left(\mathrm{PM}_{2}\right)$ corresponding to $\mathrm{GB}_{1}$, one can see how the proximity of $\mathrm{GB}_{2}$ leads to a second apparent intersection (marked with an unlabeled arrow). In Fig. 13(b) the damping has been reduced to $\lambda=-0.01$ and the spiraling of $\mathrm{W}^{\mathrm{u}}\left(\mathrm{PM}_{1}\right)$, which reflects the spiraling of $\mathrm{W}^{\mathrm{u}}\left(\mathrm{PM}_{1}\right) \cap \mathscr{P}_{1}$ toward the PM sink within $\mathscr{P}_{1}$, is correspondingly more pronounced. For these parameters, $\mathrm{W}^{\mathrm{u}}\left(\mathrm{PM}_{1}\right)$ and $\mathrm{W}^{\mathrm{s}}\left(\mathrm{PM}_{2}\right)$ do not intersect, although $\mathrm{W}^{\mathrm{s}}\left(\mathrm{PM}_{2}\right)$ comes close in two places (again marked with arrows). In Fig. 13(c) we decrease the damping still further to $\lambda=-0.003$. The spiraling part of $W^{u}\left(\mathrm{PM}_{1}\right)$ is now quite dense in comparison with Fig. 13(a) and there exist four points of approximate intersection with $\mathrm{W}^{\mathrm{s}}\left(\mathrm{PM}_{2}\right)$.

From Fig. 13(a)-(c) the trend as $\lambda \rightarrow 0$ is clear. The pancakelike surface containing the spiraling part of $\mathrm{W}^{\mathrm{u}}\left(\mathrm{PM}_{1}\right)$ will become ever more dense with spirals while it continues to be "punctured" in several places by $\mathrm{W}^{\mathrm{s}}\left(\mathrm{PM}_{2}\right)$. As $\lambda$ decreases to infinitesimal values, similarly infinitesimal changes in the parameter $f$ will be sufficient to adjust the location of $\mathrm{W}^{\mathrm{s}}\left(\mathrm{PM}_{2}\right)$ so as to intersect (one of the spirals of) $W^{u}\left(\mathrm{PM}_{1}\right)$. In this way, Fig. 13 strongly suggests that heteroclinic connections become ever more frequent as the damping is reduced, with the set of such connections having a cascade-like structure (cf. [29]) which intersects the $f$ axis at an infinite number of points. Furthermore, such a scenario can be expected to repeat in the case of $\mathrm{N}$-pulse homoclinic or heteroclinic orbits, which helps to explain the abundance of such multi-pulse cycles found in the Appendix.

The above numerical results are closely related to a number of theoretical results obtained for similar systems, particularly for perturbations of integrable two-degree-of-freedom "resonant"
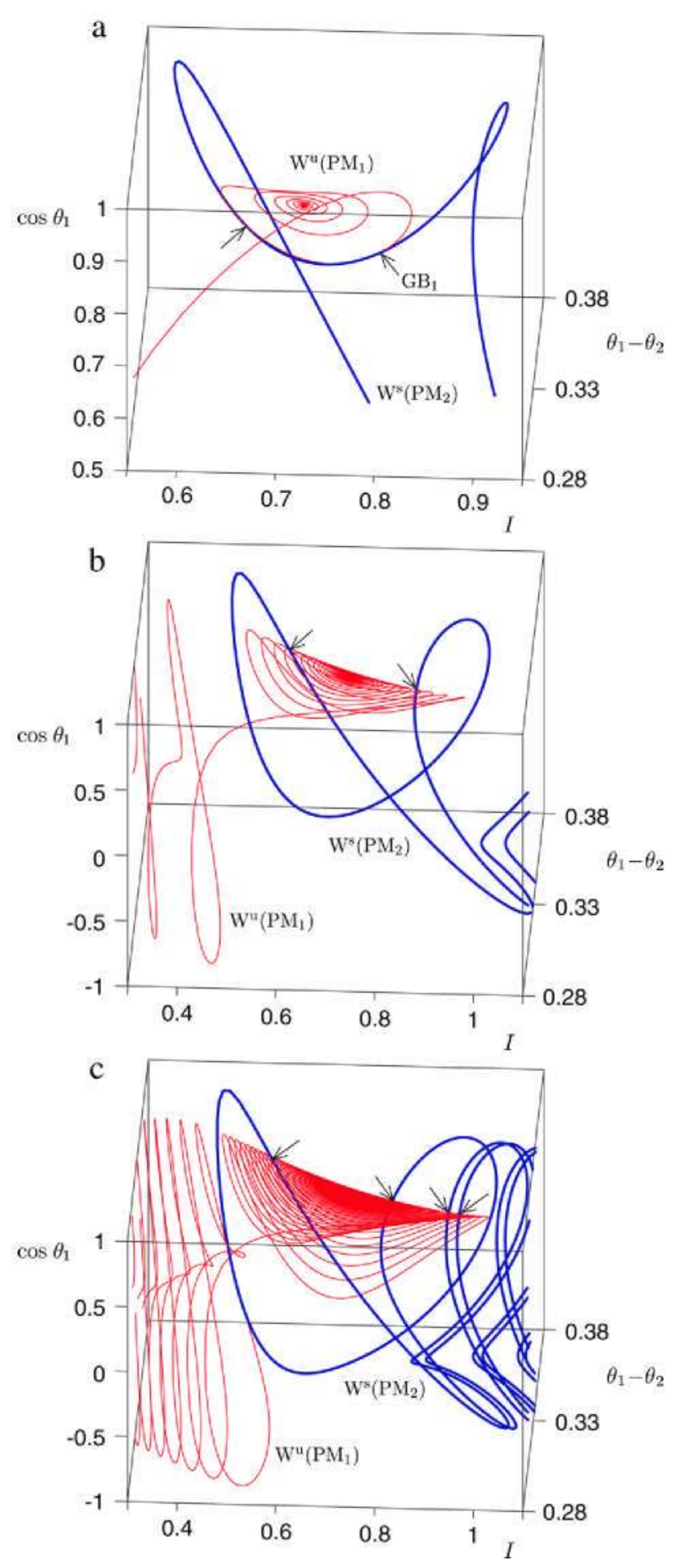

Fig. 13. Intersections of $W^{\mathrm{u}}\left(\mathrm{PM}_{1}\right)$, the unstable manifold of the saddle $\mathrm{PM}_{1} \in \mathscr{P}_{1}$. and $W^{s}\left(\mathrm{PM}_{2}\right)$, the stable manifold of the symmetry-related saddle $\mathrm{PM}_{2} \in \mathcal{P}_{2}$, with the surface $I_{1}=I_{2}$ for $f=0.04496453$ and (a) $\lambda=-0.03$, (b) $\lambda=-0.01$, (c) $\lambda=-0.003$. Approximate intersections of $W^{\mathrm{u}}\left(\mathrm{PM}_{1}\right)$ and $\mathrm{W}^{\mathrm{s}}\left(\mathrm{PM}_{2}\right)$ are indicated with an arrow; in case (a) one of these, representing $\mathrm{CB}_{1}$, is nearly exact.

Hamiltonian systems (see, e.g., [29-35]), such as system (2) with small forcing and damping. As already mentioned the unperturbed system is characterized by three-dimensional manifolds of heteroclinic connections between symmetry-related invariant manifolds $\left(\mathscr{P}_{1}\right.$ and $\left.\mathscr{P}_{2}\right)$. As such, it seems a natural candidate for the application of Melnikov-type methods, or the general energyphase method of Haller and Wiggins $[29,31]$. In particular, these 
authors showed that these types of Hamiltonian systems can contain structurally stable multi-pulse jumping orbits (orbits homoclinic to an invariant manifold) whose parameter set describes an infinite binary tree, simple and multi-pulse Shil'nikov orbits (homoclinic, or heteroclinic, to fixed points within the invariant manifold), and accompanying Smale horseshoes and chaotic dynamics.

Although we expect many of the results in [29,31] to apply to Eqs. (2), there are notable differences. Most previous work has focused on the case where the homoclinic or heteroclinic connections involve fixed points within the invariant twodimensional "slow" manifold having complex eigenvalues (centers in the case of purely Hamiltonian perturbations) while, in our case, the observed heteroclinic orbits involve saddles; see Fig. 10(c). The case of saddle-saddle connections was studied by McLaughlin et al. [33], who considered a two-mode truncation of a perturbed nonlinear Schrödinger equation. The system studied there has much in common with system (2), but lacks the interchange symmetry $\gamma$ (more specifically, their two-mode model has $\mathbf{Z}_{2} \times \mathbf{S}_{1}$ symmetry, not $\left.\mathbf{D}_{4}\right)$. Melnikov methods, combined with singular perturbation theory, were used by these authors to derive conditions for which homoclinic orbits to these saddles persist under perturbation.

Another difficulty that prevents us from applying previous results more directly is that our unperturbed connections are heteroclinic. Although the heteroclinic case does arise in the truncated nonlinear Schrödinger system (see, e.g., case (d) of Figure 6.1 in [30]) it occurs only for a particular value of $I$, and is correspondingly neglected in favor of the more generic homoclinic case. Eqs. (12), in contrast, which describe transverse connections in the unperturbed version of system (2), can be rescaled to eliminate $I$, and invariably contain heteroclinic, not homoclinic, orbits. Although the heteroclinic case has also been subjected to the energy-phase method in [32], the existing treatment assumes a purely Hamiltonian perturbation and, in addition, that the integrable limit contains a band of fixed points within the slow manifold; in contrast, the system (2) has an isolated circle of equilibria in this limit, as indicated in Fig. 10(a).

We do not attempt here to prove any rigorous results on the persistence of heteroclinic cycles in the forced damped system (2), but instead turn in the following section to an analysis of some of the global bifurcations identified numerically.

\section{Analysis of the heteroclinic bifurcation}

Here we consider an isolated global bifurcation $\left(\mathrm{GB}_{1}\right.$ or $\left.\mathrm{GB}_{2}\right)$ and show that the emergence of the figure- 8 and star periodic orbits from this bifurcation (see Fig. 5) can be understood as the result of a type of "gluing bifurcation"-perhaps more appropriately called a "symmetry-switching" bifurcation (see [36]) since both periodic orbits have a discrete symmetry $\left(\mathbf{Z}_{2}\right.$ for the figure- 8 orbit and $\mathbf{Z}_{4}$ for the star orbit).

In the neighborhood of a saddle PM the linearized flow can be written as

$\dot{x}=\lambda_{5} x, \quad \dot{y}=\lambda_{u} y, \quad \dot{u}=\lambda_{1} u, \quad \dot{v}=\lambda_{2} v$,

where $(u, v)$ are Cartesian coordinates in the invariant subspace $\left(\mathscr{P}_{1}\right.$ or $\left.\mathscr{P}_{2}\right)$ and $(x, y)$ are the transverse coordinates. The eigenvalues, as in Section 3 , are taken to be real with $\lambda_{s}, \lambda_{2}<$ 0 and $\lambda_{u}, \lambda_{1}>0$. We may then define two pairs of Poincaré sections through which generic trajectories visiting the PM must, respectively, enter and exit:

$\Sigma_{x}^{ \pm}=\{(x, y, u, v)|| x|= \pm \epsilon| y,|\leq \epsilon| u,|\leq \delta| v \mid, \leq \delta\}$,

$\Sigma_{y}^{ \pm}=\{(x, y, u, v)|| x|\leq \epsilon, y= \pm \epsilon| u,|\leq \delta| v \mid, \leq \delta\}$.
Here $\epsilon$ and $\delta$ are small parameters, which we make distinct to allow for $\epsilon \ll \delta$ in the case (occurring in Section 3) where trajectories leave the PM tangent to the invariant subspace.

The local map $\mathrm{T}_{\mathrm{PM}}: \Sigma_{x}^{ \pm} \rightarrow \Sigma_{y}^{ \pm}$is given by

$\mathrm{T}_{\mathrm{PM}}: \Sigma_{x}^{ \pm} \rightarrow \Sigma_{y}^{ \pm}:\left(\begin{array}{c}x \\ y \\ u \\ v\end{array}\right) \longmapsto\left(\begin{array}{c}\operatorname{sign}(x) \epsilon|y / \epsilon|^{-\lambda_{s} / \lambda_{u}} \\ \operatorname{sign}(y) \epsilon \\ u|y / \epsilon|^{-\lambda_{1} / \lambda_{u}} \\ v|y / \epsilon|^{-\lambda_{2} / \lambda_{U}}\end{array}\right)$.

To construct a global map we linearize about the heteroclinic connection that is assumed to exist (at $\mu=0$, say) between $\mathrm{PM}_{1} \in \mathscr{P}_{1}$ and $\rho \cdot \mathrm{PM}_{1}=\mathrm{PM}_{2} \in \mathscr{P}_{2}$. For convenience, we absorb the symmetry $\rho$ into the definition of the local coordinates so that a point $(x, y, u, v)$ near $\mathrm{PM}_{1}$ is mapped by $\rho$ to the same point in the corresponding neighborhood of $\mathrm{PM}_{2}$. In contrast, a point $(x, y, u, v)$ near $\mathrm{PM}_{1}$ is mapped by $\gamma$ to the point $(-x,-y, u, v)$ near $\mathrm{PM}_{2}$. Furthermore and without loss of generality, we assume that the heteroclinic connection from $\mathrm{PM}_{1}$ to $\mathrm{PM}_{2}$ exits the neighborhood of $\mathrm{PM}_{1}$ through $\Sigma_{y}^{+}$at the point $\mathrm{W}^{\mathrm{u}}\left(\mathrm{PM}_{1}\right) \cap \mathrm{W}^{\mathrm{s}}\left(\mathrm{PM}_{2}\right) \cap \Sigma_{y}^{+} \equiv\left(0, \epsilon, \bar{u}_{h}, 0\right)$, and that it subsequently arrives near $\mathrm{PM}_{2}$ via $\mathrm{W}^{\mathrm{u}}\left(\mathrm{PM}_{1}\right) \cap \mathrm{W}^{\mathrm{s}}\left(\mathrm{PM}_{2}\right) \cap \Sigma_{x}^{\sigma} \equiv\left(\sigma \epsilon, 0,0, \bar{v}_{h}\right)$. Thus, the heteroclinic connection at $\mu=0$ is parametrized by $\sigma= \pm 1$ and the two real constants $\bar{u}_{h}$ and $\bar{v}_{h}$. The global map may then be written as

$\mathrm{T}_{\mathrm{G}}: \Sigma_{y}^{+} \rightarrow \Sigma_{x}^{\sigma}:\left(\begin{array}{l}x \\ \epsilon \\ u \\ v\end{array}\right) \longmapsto\left(\begin{array}{c}a_{1} x+a_{2}\left(u-\bar{u}_{h}\right)+a_{3} v+\mu \\ a_{4} x+a_{5}\left(u-\bar{u}_{h}\right)+a_{6} v+r_{1} \mu \\ \bar{v}_{h}+a_{7} x+a_{8}\left(u-\bar{u}_{h}\right)+a_{9} v+r_{2} \mu\end{array}\right)$,

where the $a_{i}$ and $r_{i}$ are real constants, determined by the global flow.

A star periodic orbit exists if there is a point $(\sigma \epsilon, y, u, v)$ with $y>0$ near $\mathrm{PM}_{1}$ that is mapped by $\mathrm{T}_{\mathrm{G}} \cdot \mathrm{T}_{\mathrm{PM}}$ to $(\sigma \epsilon, y, u, v)$. (Recall that a star periodic orbit is symmetric under $\rho$.) A figure- 8 orbit, on the other hand, exists if $\mathrm{T}_{\mathrm{G}} \cdot \mathrm{T}_{\mathrm{PM}} \cdot(-\sigma \epsilon, y, u, v)=(\sigma \epsilon,-y, u, v)$, again for $y>0$. (Recall that a figure-8 periodic orbit is symmetric under $\gamma$.) Both of these conditions lead to the set of equations (plus sign for star, minus sign for figure-8):

$\pm y= \pm \sigma \epsilon a_{1}|y / \epsilon|^{\eta_{1}}+a_{2}\left(u|y / \epsilon|^{-\eta_{2}}-\bar{u}_{h}\right)+a_{3} v|y / \epsilon|^{\eta_{3}}+\mu$,

$u= \pm \sigma \epsilon a_{4}|y / \epsilon|^{\eta_{1}}+a_{5}\left(u|y / \epsilon|^{-\eta_{2}}-\bar{u}_{h}\right)+a_{6} v|y / \epsilon|^{\eta_{3}}+r_{1} \mu$,

$v= \pm \sigma \epsilon a_{7}|y / \epsilon|^{\eta_{1}}+a_{8}\left(u|y / \epsilon|^{-\eta_{2}}-\bar{u}_{h}\right)+a_{9} v|y / \epsilon|^{\eta_{3}}+\bar{v}_{h}+r_{2} \mu$,

where $\eta_{1} \equiv-\lambda_{s} / \lambda_{u}, \eta_{2} \equiv \lambda_{1} / \lambda_{u}$, and $\eta_{3} \equiv-\lambda_{2} / \lambda_{u}$. Since we are interested in solutions that satisfy $(y, u, v) \rightarrow\left(0,0, \bar{v}_{h}\right)$ as $\mu \rightarrow 0$ we must have, at leading order,

$u=\bar{u}_{h}|y / \epsilon|^{\eta_{2}}, \quad v=\bar{v}_{h}$.

Solving (23b) for $\left(u|y / \epsilon|^{-\eta_{2}}-\bar{u}_{h}\right)$ and substituting this and Eq. (24) into Eq. (23a) yields

$\tilde{\mu} \pm y= \pm A_{1}|y|^{\eta_{1}}+A_{2}|y|^{\eta_{2}}+A_{3}|y|^{\eta_{3}}$,

where $\tilde{\mu}=\left(1-r_{1} a_{2} / a_{5}\right) \mu, A_{1}=\sigma\left(a_{1}-a_{2} a_{4} / a_{5}\right) \epsilon^{1-\eta_{1}}$, $A_{2}=a_{2} \bar{u}_{h} \epsilon^{-\eta_{2}} / a_{5}$, and $A_{3}=\left(a_{3}-a_{2} a_{6} / a_{5}\right) \bar{v}_{h} \epsilon^{-\eta_{3}}$. The solutions of Eq. (25) can be grouped into three cases depending on the eigenvalue ratios $\eta_{j}$. If $\eta_{1}, \eta_{2}, \eta_{3}>1$ then star (figure-8) periodic orbits exist for $\tilde{\mu}<0(\tilde{\mu}>0)$. If $\eta_{1}<\eta_{2}, \eta_{3}, 1$ then star (figure-8) periodic orbits exist for $\tilde{\mu} A_{1}>0\left(\tilde{\mu} A_{1}<0\right)$. Finally, if $\eta_{j}<\eta_{1}, 1$ for $j=2$ or $j=3$ (or both) then star and figure- 8 periodic orbits both exist for $\tilde{\mu} A_{j}>0$. For the parameter values considered in Section 3, we have $\lambda_{1}=0.331, \lambda_{2}=-0.391, \lambda_{s}=-0.87, \lambda_{u}=$ 0.81 , giving $\eta_{1}=1.074, \eta_{2}=0.409, \eta_{3}=0.483$. Thus, the final case applies and we expect both star and figure- 8 periodic orbits on the same side of the global bifurcation. This is consistent with Fig. 5.

We do not pursue a stability analysis for these periodic orbits because the result is easily anticipated, and quite apparent from the numerics: they are unstable. This is expected because the 

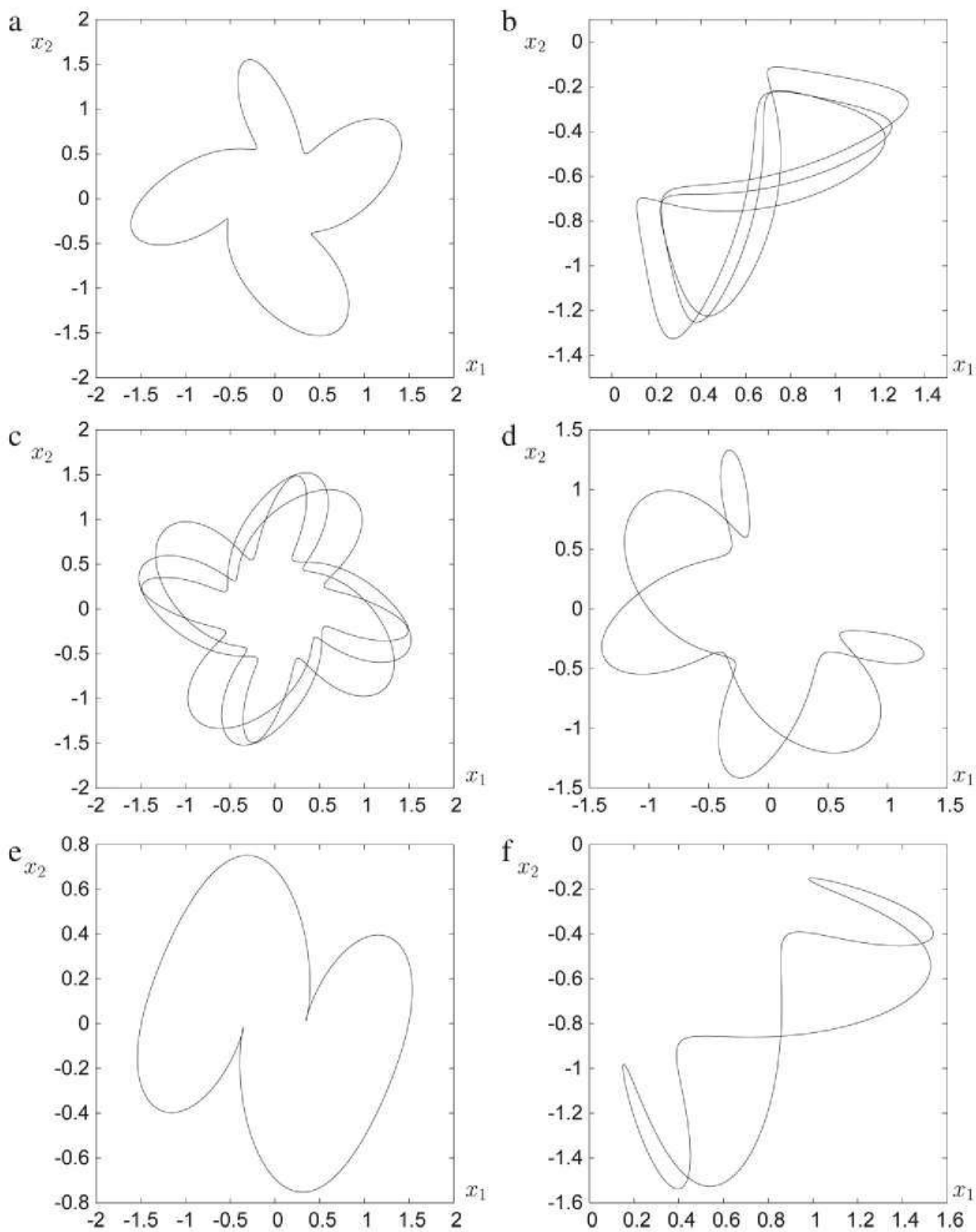

Fig. 14. (a) Stable periodic orbits at (a) $f=0.82$, (b) $f=0.53$, (c) $f=0.7$, (d) $f=0.51$, (e) $f=0.4$, and (f) $f=1.45$.

relevant fixed points have two (distinct) real positive eigenvalues, one of which describes perturbations transverse to the heteroclinic orbit. As a result all nearby periodic orbits will generically be unstable. This prediction accords with our numerical computations which reveal stable periodic orbits only far from the global bifurcations that create them (where the Poincaré map analysis is no longer valid).

\section{Conclusion}

We have shown that periodic orbits exist for the model derived in Feng and Sethna [11] for the Faraday system with square cross-section and single-frequency forcing. These periodic orbits correspond to quasiperiodic surface waves in the physical system. The periodic orbits are associated with heteroclinic bifurcations, which are themselves related to a continuum of heteroclinic connections belonging to the integrable Hamiltonian limit, some of which persist when small damping is present. Chaotic attractors were also found in the model equations, and these correspond to chaotic surface waves. The dynamics in the neighborhood of one of the heteroclinic bifurcations were examined in detail using an approximate Poincaré map, with the predictions showing agreement with numerical results.

The specific periodic orbits described in this paper were not found in the experiments by Simonelli and Gollub [3] near the interaction between $(3,2)$ and $(2,3)$ modes. There are several reasons why this might be the case. First, the coefficients used in the model might be incorrect due to neglect of the effects of contact line motion pinning, viscous effects along the vertical walls, and streaming flow driven in viscous boundary layers at the surface, bottom and sides. Second, the experiment, in sweeping through parameter space, might not have happened upon stable states such as the ones we compute because these coexist with other, simpler stable states and the existence of the new states was not known. Third, it is possible that the experiment was not run for times long compared to the decay time of the free streaming flow, i.e., it was 

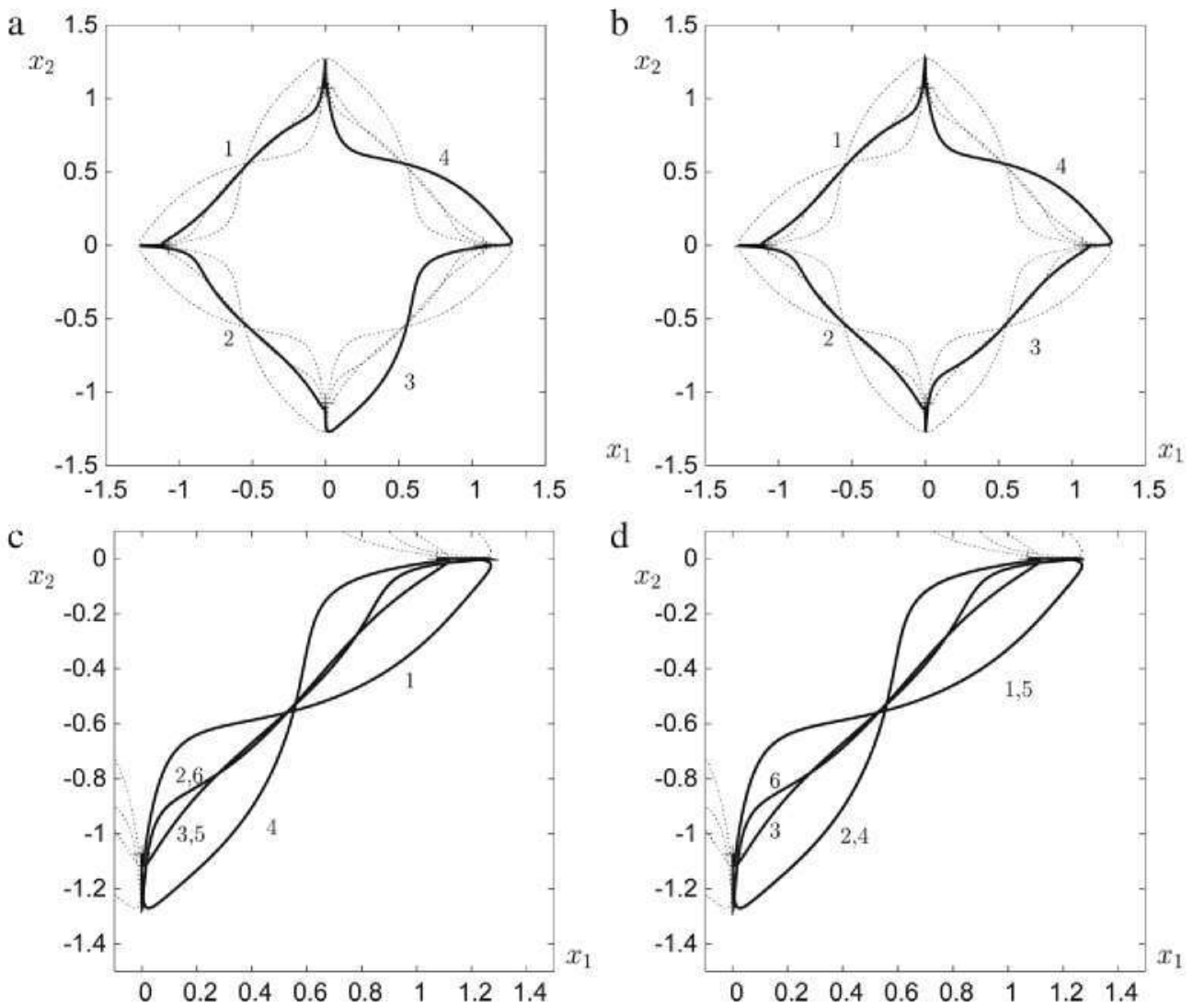

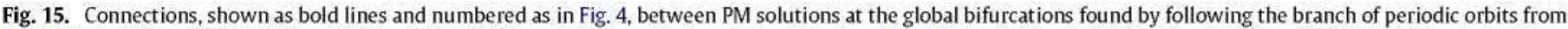

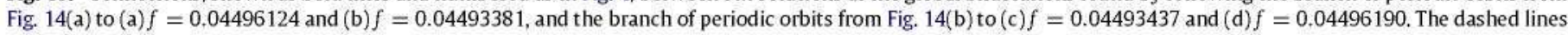
show connections which exist at $\mathrm{GB}_{1}$ and $\mathrm{GB}_{2}$.

run on time scales on which the experiment is not in fact described by Eqs. (2). This could be so because at the time the multiple time scale nature of the nearly inviscid Faraday problem was not appreciated. Finally, the observed states might be too nonlinear to be described by small amplitude theory of the type used in derivation of the model. However, in many applications this type of theory does quite well, and we note that the smaller amplitude predictions are in fact in agreement with the experiment, as shown in Fig. 1.

On the other hand, the solutions obtained here, particularly the "figure- 8 " periodic orbits, resemble one of the periodic orbits observed experimentally by Simonelli and Gollub [3], although in the experiment this appears more likely to be associated with heteroclinic connections between mixed modes instead of pure modes. This difference might be due to the neglect of an important physical effect in the coefficient computation. We remark that, if desired, one can find ad hoc coefficients for which the figure-8 periodic orbit is associated with connections between mixed mode solutions instead of the pure modes. This is a consequence of a parameter symmetry whose presence in Eqs. (2) can be established much as described for a related system in [37]. It is also possible that the solutions identified in this paper might be related to the quasiperiodic and chaotic surface waves found experimentally near onset when the container is slightly rectangular [3].

Our results suggest a great richness of possible dynamics of surface gravity-capillary waves even in the simple case of small aspect ratio containers of square cross-section with singlefrequency forcing. However, much remains to be understood about such systems, particularly with regard to the influence of container geometry, the role played by symmetry-breaking imperfections, and the coupling of the waves to the streaming flow they produce [16-18].

\section{Acknowledgments}

This work was supported in part by an Alfred P. Sloan Research Fellowship in Mathematics (JM), by the Spanish MEC under grant ESP2006-03091 (JP), and by the National Science Foundation under grant DMS-0605238 (EK).

\section{Appendix}

In this Appendix we provide a glimpse of the complexity of heteroclinic behavior present in Eqs. (2) beyond that discussed in the body of the paper. Fig. 14 shows several additional periodic orbits obtained for $A=0.1, B=-0.8, C=-1, \omega=1$, and $\lambda=-0.03$.

The branches containing the periodic orbits in Fig. 14(a)-(d) can be numerically continued in both directions to reveal (four) pairs of global bifurcations near $f=0.045$; see Figs. 15 and 16 . The connections, exact or approximate, that appear at these global bifurcations resemble the connections that exist at $\mathrm{GB}_{1}$ and $\mathrm{GB}_{2}$, suggesting that these solutions are also related to the presence of these global bifurcations.

Stable periodic solutions can also be found that, when continued in $f$, lie on isolas rather than terminating in global bifurcations. For the case of the stable periodic orbit shown in Fig. $14(\mathrm{e})$ at $f=0.4$, the corresponding solution branch exists between saddle-node bifurcations at $f=0.1227298$ and $f=$ 0.4176532 . There are symmetry-breaking bifurcations at $f=$ 0.1263139 and $f=0.3522973$ and between these values all such periodic orbits are unstable; stable limit cycles exist for the remaining values of $f$ (i.e., between saddle-node and symmetrybreaking bifurcations). Similarly, for the stable periodic orbit shown in Fig. 14(f) at $f=1.45$, the corresponding solution branch 

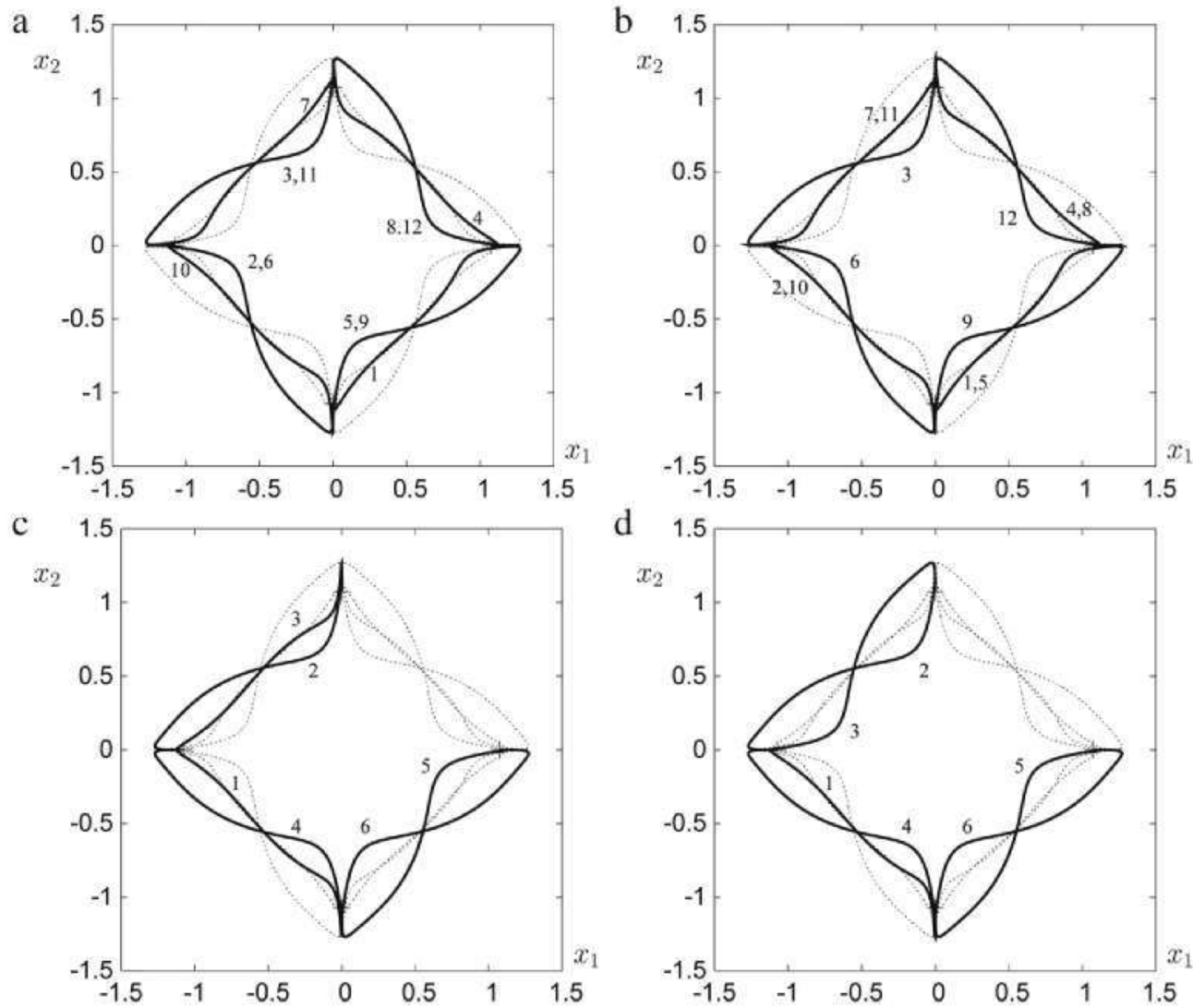

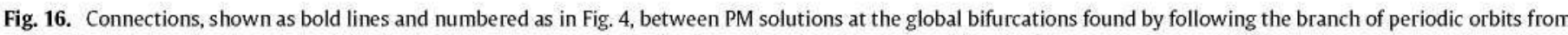

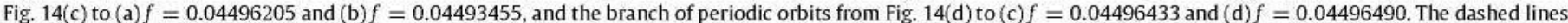
show connections which exist at $\mathrm{GB}_{1}$ and $\mathrm{GB}_{2}$.

is bounded by saddle-node bifurcations at $f=0.05273357$ and $f=1.554205$. Again two symmetry-breaking bifurcations occur, one extremely close to the lower saddle-node bifurcation, and another at $f=1.251234$. The periodic orbits are unstable between these symmetry-breaking bifurcations, while stable solutions exist for the remaining values of $f$ between the two saddle-node bifurcations.

Although we have not explored systematically this aspect of Eqs. (2) we anticipate that additional solution types are present as well.

\section{References}

[1] M. Faraday, On the forms and states assumed by fluids in contact with vibrating elastic surfaces, Phil. Trans. Roy. Soc. London 121 (1831) 319-340.

[2] S. Ciliberto, J.P. Gollub, Chaotic mode competition in parametrically forced surface waves, J. Fluid Mech. 158 (1985) 381-398.

[3] F. Simonelli, J.P. Gollub, Surface wave mode interactions: Effects of symmetry and degeneracy, J. Fluid Mech. 199 (1989) 471-494.

[4] M. Nagata, Chaotic behaviour of parametrically excited surface waves in square geometry, Eur. J. Mech, B/Fluids 10 (2 (suppl.)) (1991) 61-66.

[5] M. Umeki, Faraday resonance in rectangular geometry, J. Fluid Mech. 227 (1991) 161-192.

[6] C.M. Topaz, J. Porter, M. Silber, Multifrequency control of Faraday wave patterns, Phys. Rev. E 70 (2004) 066206.

[7] W.S. Edwards, S. Fauve, Patterns and quasi-patterns in the Faraday experiment, J. Fluid Mech. 278 (1994) 123-148.

[8] A. Kudrolli, B. Pier, J.P. Gollub, Superlattice patterns in surface waves, Physica D $123(1998)$ 99-111.

[9] H. Arbell, J. Fineberg, Temporally harmonic oscillons in Newtonian fluids, Phys. Rev. Lett. 85 (2000) 756-759.

[10] H. Arbell, J. Fineberg, Pattern formation in two-frequency forced parametric waves, Phys. Rev. E 65 (2002) 036224.

[11] Z.C. Feng, P.R. Sethna, Symmetry-breaking bifurcations in resonant surface waves, J. Fluid Mech. 199 (1989) 495-518.

[12] J.W. Miles, Surface wave damping in closed basins, Proc. Roy. Soc. London A 297 (1967) 459-475.
[13] D. Armbruster, J. Guckenheimer, S. Kim, Resonant surface waves in a square container, in: M. Singer (Ed.), Differential Equations and Computer Algebra, Academic Press, London, 1991, pp. 61-76.

[14] H. Riecke, M. Silber, L. Kramer, Temporal forcing of small-amplitude waves in anisotropic systems, Phys. Rev. E 49 (1994) 4100-4113.

[15] J. Moehlis, E. Knobloch, Wrinkled tori and bursts due to resonant tempora forcing, Physica D 151 (2001) 99-124.

[16] M. Higuera, J. Vega, E. Knobloch, Coupled amplitude-streaming flow equations for nearly inviscid Faraday waves in small aspect ratio containers, J. Nonlinear Sci. 12 (2002) 1432-1467.

[17] M. Higuera, E. Knobloch, J. Vega, Dynamics of nearly inviscid Faraday waves in almost circular containers, Physica D 201 (2005) 83-120

[18] M. Higuera, E. Knobloch, Nearly inviscid Faraday waves in slightly rectangular containers, Prog. Theor. Phys. Suppl. 161 (2006) 53-67.

[19] C.M. Topaz, M.Silber, Resonances and superlattice pattern stabilization in twofrequency forced Faraday waves, Physica D 172 (2002) 1-29.

[20] J.D. Crawford, Normal forms for driven surface waves: Boundary conditions, symmetry, and genericity. Physica D 52 (1991) 429-457.

[21] E. Doedel, A. Champneys, T. Fairgrieve, Y. Kuznetsov, B. Sandstede, X. Wang Auto 97: Continuation and bifurcation software for ordinary differential equations, 1997. Available from http://indy.cs.concordia.ca/auto/.

[22] J.W. Swift, K. Wiesenfeld, Suppression of period doubling in symmetric systems, Phys. Rev. Lett. 52 (1984) 705-708.

[23] S. Abreu, P. Aston, I. Melbourne, Symmetric chaos in a local codimension two bifurcation with the symmetry group of a square, SIAM J. Appl. Dyn. Syst. 4 (2005) $32-52$.

[24] G. Haller, Chaos Near Resonance, Springer-Verlag, New York, 1999.

[25] Z.C. Feng, P.R. Sethna, Global bifurcations in the motion of parametrically excited thin plates, Nonlinear Dynam. 4 (1993) 389-408.

[26] J.A. Zufiria, Oscillatory spatially periodic weakly nonlinear gravity waves on deep water, J. Fluid Mech. 191 (1988) 341-372.

[27] J. Guckenheimer, A. Mahalov, Instability induced by symmetry reduction, Phys. Rev. Lett. 68 (1992) 2257-2260.

[28] S. Wiggins, Global Bifurcations and Chaos: Analytical Methods, SpringerVerlag, New York, 1988.

[29] G. Haller, S. Wiggins, N-pulse homoclinic orbits in perturbations of resonant Hamiltonian system, Arch. Ration. Mech. Anal. 130 (1995) 25-101.

[30] G. Kovačič, S. Wiggins, Orbits homoclinic to resonances, with an application to chaos in a model of the forced and damped sine-Gordon equation, Physica D 57 (1992) $185-225$. 
[31] 6. Haller, 5. Wiggins, Multi-pulse jumping orbits and homoclinic trees in a model truncation of the damped-forced nonlinear Schrödinger equation. Physica D 85 (1995) 311-347.

[32] G. Haller, S. Wiggins, Geometry and chaos near resonant equilibria of 3-DOF Hamiltonian systems, Physica D 90 (1996) 319-365.

[33] D.W. McLaughlin, E.A. Overman, S. Wiggins, C. Xiong, Homoclinic orbits in a four dimensional model of a perturbed NLS equation: A geometric singular perturbation study, in: Dynamics Reported, vol. 5, Springer, New York, 1996 , Pp. $190-287$
[34] E. Shlizerman, V. Rom-Kedar, Hierarchy of bifurcations in the truncated and forced nonlinear Schrödinger model. Chaos 15 (2005) 013107.

[35] E. Shlizerman, V. Rom-Kedar, Three types of chaos in the forced nonlinear Schrödinger equation, Phys. Rev. Lett. 96 (2006) 024104.

136] M. Higuera, J. Porter, E. Knobloch. Heteroclinic dynamics in the nonlocal parametrically driven nonlinear Schrödinger equation, Physica D 162 (2002) $155-187$

[37] ]. Moehlis, E. Knobloch, Bursts in oscillatory systems with broken $D_{4}$ symmetry, Physica D 135 (2000) 263-304. 